\title{
Sur la dimension cohomologique des pro- $p$-extensions des corps de nombres
}

\author{
par CHRISTIAN MAIRE
}

À Georges Gras, pour son soixantième anniversaire
en témoignage de ma très grande reconnaissance

RÉSUMÉ. Dans ce papier, nous étudions la dimension cohomologique des groupes $G_{S}:=\mathrm{Gal}\left(\mathrm{K}_{S} / \mathrm{K}\right)$, où $\mathrm{K}_{S}$ est la pro-p-extension d'un corps de nombres $K$, non-ramifiée en dehors d'un ensemble fini de places $S$ de $\mathrm{K}$, et maximale pour ces propriétés. Si cette dimension est bien connue lorsque $S$ contient toutes les places au-dessus de $p$, elle le semble moins bien dès lors que $S$ ne contient pas toutes ces places. Néanmoins, il est possible d'obtenir des informations quand une $\mathbb{Z}_{p}$-extension $\mathrm{K}_{\infty} / \mathrm{K}$ se trouve dans $\mathrm{K}_{S} / \mathrm{K}$. En effet, dans ce cas, une étude du $\mathbb{Z}_{p}\left[\left[\mathrm{Gal}\left(\mathrm{K}_{\infty} / \mathrm{K}\right)\right]\right]$ module $\mathrm{Gal}\left(\mathrm{K}_{S} / \mathrm{K}_{\infty}\right)^{a b}$ permet de donner des conditions suffisantes pour que le pro-p-groupe $\operatorname{Gal}\left(\mathrm{K}_{S} / \mathrm{K}_{\infty}\right)$ soit libre. Si tel est le cas, il s'en déduit que la dimension de $G_{S}$ est au plus 2 . Ici, nous développons une démarche explicite mettant en jeu ces conditions afin de trouver des exemples numériques pour lesquels il est effectivement possible de calculer cette dimension cohomologique.

ABStRACT. In this paper, we study the cohomological dimension of groups $G_{S}:=\operatorname{Gal}\left(\mathrm{K}_{S} / \mathrm{K}\right)$, where $\mathrm{K}_{S}$ is the maximal pro- $p$-extension of a number field $\mathrm{K}$, unramified outside a finite set $S$ of places of K. This dimension is well-understood only when $S$ contains all places above $p$; in the case where only some of the places above $p$ are contained in $S$, one can still obtain some results if $\mathrm{K}_{S} / \mathrm{K}$ contains at least one $\mathbb{Z}_{p}$-extension $\mathrm{K}_{\infty} / \mathrm{K}$. Indeed, in that case, the study of the $\mathbb{Z}_{p}\left[\left[\mathrm{Gal}\left(\mathrm{K}_{\infty} / \mathrm{K}\right)\right]\right]-$ module $\mathrm{Gal}\left(\mathrm{K}_{S} / \mathrm{K}_{\infty}\right)^{a b}$ allows one to give sufficient conditions for the pro$p$-group $\operatorname{Gal}\left(\mathrm{K}_{S} / \mathrm{K}_{\infty}\right)$ to be free. Under the latter condition, the dimension of $G_{S}$ is at most 2. Here, we develop an explicit strategy for realizing these conditions so as to produce numerical examples for which we effectively compute this cohomological dimension. 


\section{Introduction}

La nature des pro-p-extensions à ramification restreinte d'un corps de nombres $\mathrm{K}$ semble complètement différente suivant la quantité de ramification sauvage que l'on autorise. Par exemple, si $S$ est un ensemble fini de places de $\mathrm{K}$ ne contenant aucune place au-dessus de $p$, la conjecture de Fontaine-Mazur (cf. [5], [3], [4], ...) indique que toute représentation continue $\rho: G_{S} \rightarrow \mathrm{Gl}_{m}\left(\mathbb{Z}_{p}\right)$ a une image finie, où $G_{S}=\mathrm{Gal}\left(\mathrm{K}_{S} / \mathrm{K}\right), \mathrm{K}_{S}$ étant donc la pro-p-extension maximale de K non-ramifiée en dehors de $S$. Alors qu'au contraire, si $S$ contient toutes les places au-dessus de $p$, l'extension cyclotomique montre que $G_{S}$ a au moins un quotient $p$-analytique infini. Autre différence. Quand $S$ contient toutes les places au-dessus de $p>2$, il est bien connu que la dimension cohomologique de $G_{S}$ est au plus 2 (cf. [11], [23], ... et pour $p=2$ cf. [25]). Alors que finalement quand $S$ ne contient aucune place au-dessus de $p$, peu de résultats ont été établis. Le plus remarquable est certainement celui de Labute [19]. Il donne les premiers exemples de groupes $G_{S}$ à dimension cohomologique finie. Signalons une généralisation par Schmidt [26] du résultat de Labute ainsi qu'une étude numérique de Boston [2]. Enfin, notons que quand $S$ contient quelques places au-dessus de $p$, le groupe $G_{S}$ peut-être pro-p-libre. Ce cas a été étudié par Movahhedi [21], Movahhedi et Nguyen Quang Do [22], Jaulent et Nguyen Quang Do [16], Jaulent et Sauzet [17], ...

Plaçons nous dans la situation où il existe une représentation continue $\rho: G_{S} \rightarrow \mathbb{Z}_{p}$. Posons $\mathrm{K}_{\infty}:=\mathrm{K}_{S}^{\mathrm{ker} \rho}$. L'extension $\mathrm{K}_{\infty} / \mathrm{K}$ est une $\mathbb{Z}_{p^{-}}$ extension. Il alors est possible de donner des conditions portant sur le $\mathbb{Z}_{p}[[X]]$-module $\mathcal{X}_{S}:=\operatorname{Gal}\left(\mathrm{K}_{S} / \mathrm{K}_{\infty}\right)^{a b}$ pour que le groupe $\mathrm{Gal}\left(\mathrm{K}_{S} / \mathrm{K}_{\infty}\right)$ soit pro- $p$-libre impliquant donc dans ce cas que la dimension du groupe $\operatorname{Gal}\left(\mathrm{K}_{S} / \mathrm{K}\right)$ est au plus 2. C'est ainsi que Wingberg, dans [32], a montré que si $r_{1}+r_{2}=\sum_{\mathfrak{p} \in S}\left[\mathrm{~K}_{p}: \mathbb{Q}_{p}\right]$ et que si l'invariant $\mu_{S}$ du $\mathbb{Z}_{p}\left[\left[\mathrm{Gal}\left(\mathrm{K}_{\infty} / \mathrm{K}\right)\right]\right]-$ module $\mathcal{X}_{S}$ est trivial, alors $\mathrm{Gal}\left(\mathrm{K}_{S} / \mathrm{K}_{\infty}\right)$ est libre, $\left(r_{1}, r_{2}\right)$ étant la signature du corps K. Notons que la première condition est extrêmement forte, elle appauvrit le groupe $G_{S}$ puisque, dans cette situation, le $\mathbb{Z}_{p}[[X]]$-rang de $\mathcal{X}_{S}$ est trivial (ou encore, l'invariant $\rho_{S}$ de $\mathcal{X}_{S}$ est trivial).

Le but de ce papier est la mise en place d'une stratégie permettant de trouver des exemples où le groupe $G_{S}$ est assez gros et est de dimension cohomologique finie (en fait, au plus 2). En particulier, nous voulons que le $\mathbb{Z}_{p}[[X]]$-rang du module $\mathcal{X}_{S}$ soit non nul. 
Nous aurons ainsi en permanence à l'esprit l'idée de rendre la démarche la plus explicite possible. Ceci nous permettra de détailler deux exemples pour lesquels

(i) $G_{S}$ est de dimension cohomologique 2 (et $S \subsetneq S_{p}$ );

(ii) il existe une suite exacte

$$
1 \longrightarrow H_{S} \longrightarrow G_{S} \longrightarrow \mathbb{Z}_{p} \longrightarrow 1
$$

où $H_{S}^{a b}$ s'injecte avec un conoyau fini dans $\mathbb{Z}_{p}[[X]]^{\rho_{S}}$, avec $\rho_{S} \geq 1$.

Reprenons succintement les points clefs de notre méthode. Essentiellement tout tourne autour de l'invariant $\mu_{S}$. Nous voulons obtenir des situations où le groupe $\operatorname{Gal}\left(\mathrm{K}_{S} / \mathrm{K}_{\infty}\right)$ est libre. Pour ce faire, il suffit de vérifier que d'une part une certaine condition arithmétique est satisfaite pour le corps $\mathrm{K}$ et que d'autre part l'invariant $\mu_{S}$ est trivial. Le premier point est aisé à tester numériquement. Quant au second, il peut être vérifié après quelques calculs dans la $\mathbb{Z}_{p}$-extension $\mathrm{K}_{\infty} / \mathrm{K}$. Mais pour ce faire, il est nécessaire de déterminer explicitement les étages de celle-ci. Ceci se fera à l'aide d'un logarithme $p$-adique construit sur les idéaux de K, utilisant ainsi des travaux de Gras [7], [8], [9]. Ensuite, pour vérifier la trivialité de $\mu_{S}$, on est tenté d'utiliser le lemme de Nakayama. Malheureusement, une application brutale de ce lemme au module $\mathcal{X}_{S}$ tue les invariants d'Iwasawa $\rho_{S}$ et $\mu_{S}$ simultanément, appauvrissant donc le groupe $G_{S}$. Pour remédier à ceci, et c'est le second point clef de notre méthode, nous appliquerons le lemme de Nakayama sur la partie de torsion du $\mathbb{Z}_{p}[[X]]$-module $\mathcal{X}_{S}$. Il faudra alors s'assurer qu'une certaine condition arithmétique est satisfaite le long de $\mathrm{K}_{\infty} / \mathrm{K}$. Ce sont des résultats de dualité dans $\mathrm{K}_{\infty} / \mathrm{K}$ qui permettront de vérifier cette condition et ce, après un nombre fini de calculs.

Dans la section 1, nous introduisons les principales notations. Dans la section 2, nous rappellerons les résultats standards de cohomologie des pro- $p$-groupes ainsi que ceux de théorie d'Iwasawa dont nous aurons besoin. Dans la section 3, nous développerons les notions de $S$-conditions de type "Leopoldt". Ces conditions de deux types, arithmétiques et cohomologiques, sont fondamentales dans la recherche d'exemples. La notion de $S$-condition cohomologique apporte des résultats sur la structure de $G_{S}$ tandis que la notion de $S$-condition arithmétique se prête parfaitement aux calculs. Nous nous efforcerons ainsi de trouver le maximum de liens entre celles-ci. Dans la section 4, nous présenterons les résultats nécessaires pour effectuer les calculs dans une $\mathbb{Z}_{p}$-extension, en particulier le logarithme défini par Gras. Dans la section 5 , nous présenterons des résultats de dualité le long d'une $\mathbb{Z}_{p}$-extension quelconque. Enfin, dans la section 6 , nous présenterons deux exemples. 
Pour finir, notons qu'un autre contrôle possible de la ramification sauvage est celui de la "profondeur". C'est une philosophie assez différente. Nous renvoyons à [12] pour plus détails.

L'ensemble des calculs ont été effectués avec GP-PARI [1].

\section{Remerciements.}

Ce travail a vu le jour lors de mon passage dans la chaire de Structures Algébriques et Géométriques de l'EPF Lausanne. Il a été terminé lors de ma visite à University of Massachussets, Amherst. Je remercie Éva Bayer et Farshid Hajir pour leur chaleureux accueil.

Enfin, je tiens également à remercier Thong Nguyen Quang Do pour ses remarques à propos de ces questions de dimension cohomologique.

\section{Notations}

Fixons un corps de nombres $\mathrm{K}$ ainsi qu'un nombre premier $p$. Les objets suivants interviendront tout au long de ce papier :

- $S_{p}=\left\{\mathfrak{p} \in P l_{\mathrm{K}}, \mathfrak{p} \mid p\right\}$

- $S$ et $T$ désignerons deux ensembles disjoints de places de K. On supposera $S$ contenu dans $S_{p}$;

- $E_{\mathrm{K}}$ désignera le groupe des unités de l'anneau des entiers $\mathcal{O}_{\mathrm{K}}$ de $\mathrm{K}$; plus généralement $E_{\mathrm{K}}^{T}:=\left\{\varepsilon \in \mathcal{O}_{\mathrm{K}}, \mathrm{v}_{\mathfrak{p}}(\varepsilon)=0, \forall \mathfrak{p} \notin T\right\}$ sera le groupe des $T$-unités de $\mathcal{O}_{\mathrm{K}}$;

- $\mathrm{K}_{S}$ désignera la pro-p-extension maximale de $\mathrm{K}$, non-ramifiée en dehors de $S$ (les places réelles restent réelles); $G_{S}=\mathrm{Gal}\left(\mathrm{K}_{S} / \mathrm{K}\right)$;

- $\widetilde{\mathrm{K}_{S}}$ désignera le compositum de toutes les $\mathbb{Z}_{p}$-extensions de $\mathrm{K}$ contenues dans $\mathrm{K}_{S}$;

- $\mathrm{K}_{\infty}$ désignera une $\mathbb{Z}_{p}$-extension de $\mathrm{K}$ contenue dans $\mathrm{K}_{S} / \mathrm{K} ; H_{S}=$ $\operatorname{Gal}\left(\mathrm{K}_{S} / \mathrm{K}_{\infty}\right) ; \chi_{S}=H_{S}^{a b}=H_{S} /\left[H_{S} ; H_{S}\right]$.

- $\mathrm{K}_{\infty}$ étant fixé, $\mathrm{K}_{n}$ sera l'unique sous-corps de $\mathrm{K}_{\infty} / \mathrm{K}$ de degré $p^{n}$ sur $\mathrm{K}$

- $\delta_{S}=\sum_{\mathfrak{p} \in S}\left[K_{\mathfrak{p}}: \mathbb{Q}_{p}\right]$ désignera le degré de $S$;

- $\widetilde{S}$ désignera l'ensemble des places de $\mathrm{K}$ qui sont totalement décomposées dans $\mathrm{K}_{\infty} / \mathrm{K} ; \widetilde{s}=\# \widetilde{S}$

- $S_{n}=\left\{\mathfrak{P} \in P l_{\mathrm{K}_{n}}, \mathfrak{P} \mid \mathfrak{p}, \mathfrak{p} \in S\right\}, s_{n}=\# S_{n}$

- $\mathrm{K}_{n, S}^{T}$ désignera la pro- $p$-extension maximale de $\mathrm{K}_{n}$ non-ramifiée en dehors de $S$ et où toutes les places de $T$ sont décomposées ; $G_{n, S}^{T}=$ $\operatorname{Gal}\left(\mathrm{K}_{n, S}^{T} / \mathrm{K}\right)$; 
- $\mathrm{K}_{n, T}^{S}$ désignera la pro-p-extension maximale de $\mathrm{K}_{n}$ non-ramifiée en dehors de $T$ et où toutes les places de $S$ sont décomposées ; $G_{n, T}^{S}=$ $\operatorname{Gal}\left(\mathrm{K}_{n, T}^{S} / \mathrm{K}\right)$;

- $\mathrm{K}_{n, S}^{T, a b}$ désignera la pro-p-extension abélienne maximale de $\mathrm{K}_{n}$ contenue dans $\mathrm{K}_{S}^{T} ; G_{n, S}^{T, a b}=\operatorname{Gal}\left(\mathrm{K}_{n, S}^{T, a b} / \mathrm{K}\right)$;

- $\mathrm{K}_{n, T}^{S, a b}$ est la pro- $p$-extension abélienne maximale de $\mathrm{K}_{n}$ contenue dans $\mathrm{K}_{n, T}^{S} ; G_{n, T}^{S, a b}=\operatorname{Gal}\left(\mathrm{K}_{n, T}^{S, a b} / \mathrm{K}\right)$;

- $\mathrm{L}_{S}^{T}=\bigcup_{n} \mathrm{~K}_{n, S}^{T, a b} ; \mathcal{X}_{S}^{T}=\operatorname{Gal}\left(\mathrm{L}_{S}^{T} / \mathrm{K}_{\infty}\right)^{a b} ; \mathrm{L}_{T}^{S}=\bigcup_{n} \mathrm{~K}_{n, T}^{S, a b} ; \mathcal{X}_{T}^{S}=$ $\operatorname{Gal}\left(\mathrm{L}_{T}^{S} / \mathrm{K}_{\infty}\right)^{a b}$

- $\left(\rho_{T}^{S}, \mathrm{r}_{T}^{S}, \lambda_{T}^{S}\right)$ (respectivement $\left.\left(\rho_{S}^{T}, \mathrm{r}_{S}^{T}, \lambda_{S}^{T}\right)\right)$ désignerons les invariants d'Iwasawa du $\operatorname{Gal}\left(\mathrm{K}_{\infty} / \mathrm{K}\right)$-module $\mathcal{X}_{S}^{T}$ (resp. $\mathcal{X}_{T}^{S}$ ) (cf. section 3);

- $U_{S}^{1}=\prod_{\mathfrak{p} \in S} U_{\mathfrak{p}}^{1}$, où $U_{\mathfrak{p}}^{1}$ est le groupe des unités principales du complété $\mathrm{K}_{\mathfrak{p}}$ de $\mathrm{K}$ en $\mathfrak{p} ;$ le morphisme de $\mathbb{Z}_{p}$-modules $\varphi_{S}: \mathbb{Z}_{p} \otimes_{\mathbb{Z}} E_{\mathrm{K}} \rightarrow U_{S}^{1}$ est le morphisme naturel de plongements. Plus généralement, $U_{S_{n}}^{1}=$ $\prod_{\mathfrak{p} \in S_{n}} U_{\mathfrak{p}}^{1}$ et $\varphi_{n, S}: \mathbb{Z}_{p} \otimes_{\mathbb{Z}} E_{\mathrm{K}_{n}} \rightarrow U_{S_{n}}^{1}$. Rappelons que le $\mathbb{Z}_{p}$-rang de $G_{S}^{T, a b}$ est exactement le $\mathbb{Z}_{p}$-rang du conoyau de $\varphi_{S}^{T}: \mathbb{Z}_{p} \otimes_{\mathbb{Z}} E_{\mathrm{K}}^{T}: \rightarrow$ $\prod_{\mathfrak{p} \in S} U_{\mathfrak{p}}^{1},(\mathrm{cf} .[7], \ldots)$

- Si $A$ est un pro- $p$-groupe, on dénotera par $: d_{p} A=\operatorname{dim}_{\mathbb{F}_{p}} A / A^{p}[A ; A]$ le $p$-rang de $A /[A ; A]$, par $\operatorname{rk}_{\mathbb{Z}_{p}} A$ le $\mathbb{Z}_{p}$-rang de $A /[A ; A]$ et par $\operatorname{Tor}_{\mathbb{Z}_{p}}(A)$ la partie de torsion de $A /[A ; A]$.

- Les polynômes $\omega_{n}$ et $\omega_{m, n}$ de l'algèbre d'Iwasawa $\Lambda:=\mathbb{Z}_{p}[[X]]$ sont définis comme suit : $\omega_{n}=(1+X)^{p^{n}}-1$, et pour $m>n, \omega_{m, n}=$ $\omega_{m} / \omega_{n}$. Le polynôme $\omega_{n+1, n}$ est irréductible de degré $(p-1) p^{n}$.

- Enfin, si $\mathcal{X}$ est un $\Lambda$-module, et $f \in \Lambda, \mathcal{X}^{f}$ dénotera le sous- $\Lambda$-module de $\mathcal{X}$ constitué des éléments de $\mathcal{X}$ annulés par $f$.

\section{Rappels}

2.1. Dimension cohomologique et suite spectrale. L'ensemble des résultats de ce paragraphe sont issus de [18], [23], [29], ...

2.1.1. Rappelons, pour commencer, la notion de dimension cohomologique.

Soit $G$ un pro-p-groupe. On dit que $G$ est de dimension cohomologique $\operatorname{cd}(G)=m$, si le groupe $H^{m+1}\left(G, \mathbb{F}_{p}\right)$ est trivial et si $H^{m}\left(G, \mathbb{F}_{p}\right)$ ne l'est pas.

Pour déterminer un majorant de la dimension cohomologique d'un pro$p$-groupe, trois critères sont bien connus.

Proposition 2.1. 1) Soit $H$ un sous-groupe fermé d'un pro-p-groupe $G$. Alors $\operatorname{cd}(H) \leq \operatorname{cd}(G)$. Il y a égalité lorsque $H$ est d'indice fini et $\operatorname{cd}(G)<\infty$. 
2) Soit $H$ un sous-groupe fermé et distingué d'un pro-p-groupe $G$. Alors $\operatorname{cd}(G) \leq \operatorname{cd}(G / H)+\operatorname{cd}(H)$. Il y a égalité lorsque cd $(G / H), \operatorname{cd}(H)$ et $H^{n}\left(H, \mathbb{F}_{p}\right)$ sont finis, où $n=\operatorname{cd}(H)$.

3) Pour un pro-p-groupe $G$ et lorsque celle-ci a un sens, notons par $\chi_{n}(G)$ la caractéristique d'Euler-Poincaré tronquée à l'ordre $n$. Si cette caractéristique est multiplicative pour les sous-groupes ouverts de $G$, c'està-dire si pour tout sous-groupe ouvert $U$ de $G, \chi_{n}(U)=(G: U) \chi_{n}(G)$, alors la dimension cohomologique de $G$ est au plus $n$.

À ce stade, il convient de citer un résultat de Schmidt [25] généralisant le point 3 de la proposition précédente.

Proposition 2.2. S'il existe une constante c telle que pour tout sousgroupe ouvert $U$ d'un pro-p-groupe infini $G$, il vient : $(-1)^{n} \chi_{n}(U)+c \geq$ $(-1)^{n}(G: U) \chi_{n}(G)$, alors la dimension cohomologique de $G$ est au plus $n$.

2.1.2. Soit $\mathcal{C}$ la catégorie des groupes abéliens localement compacts. Pour un groupe $M \in \mathcal{C}$, notons par $M^{*}$ le dual de Pontryagin de $M$ : c'est le groupe $\operatorname{Hom}(M, \mathbb{R} / \mathbb{Z})$ des homomorphismes continus de $M$ vers $\mathbb{R} / \mathbb{Z}$. On rappelle que cette dualité est un foncteur contravariant et que pour $M \in \mathcal{C}$, $M \simeq\left(M^{*}\right)^{*}$. Cette dualité transforme les groupes discrets en groupes compacts, les groupes divisibles en groupes sans torsion. Lorsque $M$ est un pro-p-groupe, on a $\operatorname{Hom}(M, \mathbb{R} / \mathbb{Z}) \simeq \operatorname{Hom}\left(M, \mathbb{Q}_{p} / \mathbb{Z}_{p}\right)$.

Proposition 2.3. Soit $G$ un pro-p-groupe et $A$ un $G$-module compact. Alors, pour tout $n \geq 0$,

$$
H^{n}\left(G, A^{*}\right) \simeq H_{n}(G, A)^{*} .
$$

Pour $n=0$, cette égalité s'écrit :

$$
\left(A_{G}\right)^{*} \simeq\left(A^{*}\right)^{G} .
$$

On arrive ainsi à une conséquence bien connue du lemme de Nakayama :

Proposition 2.4. Pour $G \simeq \mathbb{Z}_{p}$ et tout $G$-module discret $A$, on a $A=0$ si et seulement si $A^{G}=0$.

Rappelons une équivalence là aussi bien connue pour caractériser la liberté d'un pro-p-groupe.

Proposition 2.5. Un pro-p-groupe non trivial $G$ est libre $(\operatorname{cd}(G)=1)$ si et seulement si, $H^{2}\left(G, \mathbb{Q}_{p} / \mathbb{Z}_{p}\right)=0$ et $G^{a b}$ est sans torsion.

Preuve. Partons de la suite exacte :

$$
1 \longrightarrow \mathbb{Z}_{p} \stackrel{p}{\longrightarrow} \mathbb{Z}_{p} \longrightarrow \mathbb{Z}_{p} / p \mathbb{Z}_{p} \longrightarrow 1
$$

qui après passage à l'homologie donne

$\cdots H_{2}\left(G, \mathbb{Z}_{p}\right) \stackrel{p}{\longrightarrow} H_{2}\left(G, \mathbb{Z}_{p}\right) \longrightarrow H_{2}\left(G, \mathbb{F}_{p}\right) \longrightarrow H_{1}\left(G, \mathbb{Z}_{p}\right) \stackrel{p}{\longrightarrow} H_{1}\left(G, \mathbb{Z}_{p}\right) \cdots$ 
ou encore

$$
H_{2}\left(G, \mathbb{Z}_{p}\right) / p \hookrightarrow H_{2}\left(G, \mathbb{F}_{p}\right) \longrightarrow G^{a b}[p] \longrightarrow 1 .
$$

Il vient ainsi $d_{p} H^{2}\left(G, \mathbb{F}_{p}\right)=d_{p} H_{2}\left(G, \mathbb{Z}_{p}\right)+d_{p} G^{a b}[p]$. L'équivalence est alors immédiate.

2.1.3. Partons de la suite exacte de pro- $p$-groupes

$$
1 \longrightarrow H \longrightarrow G \longrightarrow \Gamma \longrightarrow 1,
$$

avec $\Gamma \simeq \mathbb{Z}_{p}$

Lorsque $A$ est un $\Gamma$-module de $p$-torsion, on sait que $H^{1}(\Gamma, A) \simeq A_{\Gamma}$ (cf. par exemple [32], proposition 1.6.3, chapitre I).

Alors, la suite spectrale de Hochschild-Serre appliquée à la précédente suite exacte donne :

Proposition 2.6.

$1 \longrightarrow H^{1}\left(\Gamma, H^{1}\left(H, \mathbb{Q}_{p} / \mathbb{Z}_{p}\right)\right) \longrightarrow H^{2}\left(G, \mathbb{Q}_{p} / \mathbb{Z}_{p}\right) \longrightarrow H^{2}\left(H, \mathbb{Q}_{p} / \mathbb{Z}_{p}\right)^{\Gamma} \longrightarrow 1$, ò̀ $H^{1}\left(\Gamma, H^{1}\left(H, \mathbb{Q}_{p} / \mathbb{Z}_{p}\right)\right)$ s'identifie ̀̀ $\left(\left(H^{a b}\right)^{*}\right)_{\Gamma}$.

Ce qui permet d'en déduire le corollaire suivant :

Corollaire 2.1. La trivialité du groupe $H^{2}\left(G, \mathbb{Q}_{p} / \mathbb{Z}_{p}\right)$ implique la trivialité de $H^{2}\left(H, \mathbb{Q}_{p} / \mathbb{Z}_{p}\right)$.

Preuve. On utilise la proposition 2.6 puis la proposition 2.4 appliquée à $H^{2}\left(H, \mathbb{Q}_{p} / \mathbb{Z}_{p}\right)$.

2.2. L'algèbre d'Iwasawa. Soit $\Lambda:=\mathbb{Z}_{p}[[X]]$ l'algèbre d'Iwasawa topologiquement isomorphe à $\mathbb{Z}_{p}[[\Gamma]]:=\lim _{\leftarrow} \mathbb{Z}_{p}\left[\Gamma / \Gamma^{p^{n}}\right]$, où $\Gamma=<\gamma>\simeq \mathbb{Z}_{p}$ et où $\gamma \rightarrow X+1$. Rappelons que si $\mathcal{X}$ est un $\Lambda$-module de type fini, il existe des polynômes distingués irréductibles $f_{i} \in \mathbb{Z}_{p}[X]$, des entiers $a_{i}$, $b_{i}$, et $\rho_{\mathcal{X}}$ tels que $\mathcal{X}$ est pseudo-isomorphe à

$$
\mathcal{E}=\Lambda^{\rho_{\mathcal{X}}} \oplus \bigoplus_{i=1, \cdots, \mathrm{r}_{\mathcal{X}}} \Lambda /\left(p^{a_{i}}\right) \oplus \bigoplus_{i} \Lambda /\left(f_{i}^{b_{i}}\right)
$$

(c'est à dire, qu'il existe un morphisme de $\Lambda$-modules $\phi$ de $\mathcal{X}$ vers $\mathcal{E}$ à noyau et conoyau finis). Les entiers $\lambda_{\mathcal{X}}=\sum_{i} b_{i} \operatorname{deg}\left(f_{i}\right), \mu_{\mathcal{X}}=\sum_{i=1}^{\mathrm{r}_{\mathcal{X}}} a_{i}$ et $\rho_{\mathcal{X}}$ sont appelés invariants d'Iwasawa de $\mathcal{X}$.

Le polynôme $P_{\mathcal{X}}=\prod_{i} f_{i}^{b_{i}}$ est appelé polynôme caractéristique de $\mathcal{X}$.

Ce théorème de structure permet de donner des informations sur le comportement du $p$-rang de certains quotients de $\mathcal{X}$ le long de $\mathrm{K}_{\infty} / \mathrm{K}$.

Lemme 2.1. Soient $\mathcal{X}$ un $\Lambda$-module de type fini, pseudo-isomorphe à $\mathcal{E}$, et $\mathcal{Y}$ un sous- $\Lambda$-module de $\mathcal{X}$ tels que le $\mathbb{Z}_{p}$-module $\mathcal{X} / \mathcal{Y}$ soit de type fini. Fixons un entier $m>0$. Alors 
(i) $d_{p} \mathcal{X} / \omega_{n, m} \mathcal{Y}=p^{n}\left(\rho_{\mathcal{X}}+\mathrm{r}_{\mathcal{X}}\right)+O(1)$;

(ii) $\mathrm{rk}_{\mathbb{Z}_{p}} \mathcal{X} / \omega_{n, m} \mathcal{Y}=p^{n} \rho \mathcal{X}+O(1)$;

(iii) $\operatorname{rk}_{\mathbb{Z}_{p}} \mathcal{X} / \omega_{n} \mathcal{X}=p^{n} \rho_{\mathcal{X}}+\sum_{i} \operatorname{rk}_{\mathbb{Z}_{p}} \Lambda /\left(f_{i}, \omega_{n}\right)$

Preuve. Nous nous contenterons de montrer le point (i). Partons de la suite exacte

$$
1 \longrightarrow A \longrightarrow \mathcal{X} \stackrel{\phi}{\longrightarrow} \mathcal{E} \longrightarrow B \longrightarrow 1
$$

où $A$ et $B$ sont deux $\Lambda$-modules finis. Cette suite exacte devient

$$
\begin{aligned}
\frac{B_{0}}{\omega_{n, m} \mathcal{Y}+p \mathcal{X}} \hookrightarrow \frac{\mathcal{X}}{\omega_{n, m} \mathcal{Y}+p \mathcal{X}} \longrightarrow \frac{\mathcal{E}}{\omega_{n, m} \mathcal{E}+p \mathcal{E}} \longrightarrow \\
\longrightarrow \frac{\mathcal{E}}{\phi(\mathcal{X})+\omega_{n, m} \mathcal{E}+p \mathcal{E}} \longrightarrow 1,
\end{aligned}
$$

où $B_{0}=\phi^{-1}\left(\omega_{n, m} \mathcal{E}+p \mathcal{E}\right)$. Clairement, le dernier membre de cette suite est fini. Il reste à évaluer le premier membre. Pour cela, soit l'homomorphisme de $\Lambda$-modules

$$
\begin{aligned}
& F: B_{0} /\left(\omega_{n, m} \mathcal{Y}+p \mathcal{X}\right) \rightarrow \omega_{n, m} \mathcal{E}+p \mathcal{E} /\left(\phi\left(\omega_{n, m} \mathcal{Y}\right)+p \phi(\mathcal{X})\right) \\
& \bar{x} \mapsto \overline{\phi(x)}
\end{aligned}
$$

Alors $\operatorname{ker} F$ est exactement $\left(\omega_{n, m} \mathcal{Y}+p \mathcal{X}+\operatorname{ker} \phi\right) /\left(\omega_{n, m} \mathcal{Y}+p \mathcal{X}\right)$ et est donc fini.

Maintenant, la suite exacte

$$
1 \longrightarrow \frac{\phi(\mathcal{X})}{\phi(\mathcal{Y})} \longrightarrow \frac{\mathcal{E}}{\phi(\mathcal{Y})} \longrightarrow \frac{\mathcal{E}}{\phi(\mathcal{X})} \longrightarrow 1
$$

indique que $\mathcal{E} / \phi(\mathcal{Y})$ est de type fini sur $\mathbb{Z}_{p}$ (car $\mathcal{X} / \mathcal{Y}$ l'est). Il en est ainsi de même pour l'image de $F$ ( $F$ est surjectif). En conclusion, $d_{p} \mathcal{X} / \omega_{n, m} \mathcal{Y}=$ $d_{p} \mathcal{E} / \omega_{n, m} \mathcal{E}+O(1)$, d'où le résultat.

Lemme 2.2. Soient $\mathcal{X}$ un $\Lambda$-module de type fini, d'invariants $\left(\rho_{\mathcal{X}}, \mathrm{r}_{\mathcal{X}}, \lambda_{\mathcal{X}}\right)$, et $\mathcal{Y}$ un sous- $\Lambda$-module de $\mathcal{X}$.

1) Supposons qu'il existe deux entiers $m>n \geq 0$ tels que $\mathcal{X} / \mathcal{Y}=$ $\mathcal{X} / \omega_{m, n} \mathcal{Y}$. Alors $\mathcal{X}=\mathcal{X} / \mathcal{Y}$. En particulier, si $\mathcal{X} / \mathcal{Y}$ est de type fini sur $\mathbb{Z}_{p}$, on $a \rho_{\mathcal{X}}=\mathrm{r}_{\mathcal{X}}=0$ et $\lambda_{\mathcal{X}}=\operatorname{dim}_{\mathbb{Q}_{p}}\left(\mathbb{Q}_{p} \otimes_{\mathbb{Z}_{p}} \mathcal{X} / \mathcal{Y}\right)$.

2) Supposons qu'il existe deux entiers $m>n \geq 0$ tel que $d_{p} \mathcal{X} / \omega_{m, n} \mathcal{Y}=$ $d_{p} \mathcal{X} / \mathcal{Y}$. Alors $\mathcal{X} / p \mathcal{X}=\mathcal{X} /(p \mathcal{X}+\mathcal{Y})$. En particulier, si $\mathcal{X} / \mathcal{Y}$ est de type fini sur $\mathbb{Z}_{p}, \rho_{\mathcal{X}}=\mathrm{r}_{\mathcal{X}}=0$.

Preuve. 1) Par hypothèse $\omega_{m, n} \mathcal{Y}=\mathcal{Y}$. Comme $\omega_{m, n}$ appartient à l'idéal maximal $(p, T)$, par le lemme de Nakayama, $\mathcal{Y}=0$. Ainsi $\mathcal{X} / \mathcal{Y}=\mathcal{X}$. Lorsque $\mathcal{X} / \mathcal{Y}$ est de type fini sur $\mathbb{Z}_{p}$, le $p$-rang de $\mathcal{X}$ est alors fini et par conséquent $\mu_{\mathcal{X}}=\rho_{\mathcal{X}}=0$.

2) Par hypothèse, on a l'isomorphisme $\mathcal{X} /\left(\omega_{m, n} \mathcal{Y}+p \mathcal{X}\right) \simeq \mathcal{X} /(\mathcal{Y}+p \mathcal{X})$. Ainsi, $\omega_{m, n} \mathcal{Y}+p \mathcal{X}=\mathcal{Y}+p \mathcal{X}$. Par le lemme de Nakayama, le $\Lambda$-module 
$(\mathcal{Y}+p \mathcal{X}) / p \mathcal{X}$ est trivial et ainsi $\mathcal{Y} \subset p \mathcal{X}$. Par conséquent, $\mathcal{X} /(\mathcal{Y}+p \mathcal{X})=$ $\mathcal{X} / p \mathcal{X}$. Lorsque $\mathcal{X} / \mathcal{Y}$ est de type fini sur $\mathbb{Z}_{p}$, on trouve bien $\mu_{\mathcal{X}}=\rho_{\mathcal{X}}=0$.

2.2.1. Modules d'Iwasawa et dimension cohomologique. Soient $G$ un pro$p$-groupe et, $H$ un sous-groupe fermé et distingué de $G$ vérifiant $G / H=$ $\Gamma \simeq \mathbb{Z}_{p}$. Rappelons que $\mathcal{X}=H^{a b}$ est un $\Lambda$-module (par rapport à $\Gamma$ ).

Lemme 2.3. Lorsque $H^{2}\left(G, \mathbb{Q}_{p} / \mathbb{Z}_{p}\right)$ est trivial, $\mathcal{X}$ n'a pas de sous-module fini.

Preuve. Puisque $H^{2}\left(G, \mathbb{Q}_{p} / \mathbb{Z}_{p}\right)$ est trivial, la proposition 2.6 indique que $(\mathcal{X})^{\Gamma}$ est trivial. Ainsi, le sous-module $A^{\Gamma}$ des invariants par $\Gamma$ d'un sousmodule fini $A$ de $\mathcal{X}$ est trivial, ce qui implique que $A$ est trivial (d'après la proposition 2.4).

Cette proposition permet alors de montrer la proposition suivante :

Proposition 2.7. Sous les conditions $\mathrm{r}_{\mathcal{X}}=0$ et $H^{2}\left(G, \mathbb{Q}_{p} / \mathbb{Z}_{p}\right)=0$, le pro-p-groupe $H$ est libre. Ainsi, $\operatorname{cd}_{p}(G) \leq 2$.

Preuve. Supposons $H^{2}\left(G, \mathbb{Q}_{p} / \mathbb{Z}_{p}\right)$ trivial. D'après le corollaire 2.1, nous avons $H^{2}\left(H, \mathbb{Q}_{p} / \mathbb{Z}_{p}\right)=0$. D'autre part, d'après le lemme précédent, $\mathcal{X}=$ $H^{a b}$ n'a pas de sous-module fini. Ainsi

$$
\mathcal{X} \hookrightarrow \Lambda^{\rho_{\mathcal{X}}} \oplus \bigoplus_{i=1, \cdots, \mathrm{r}_{\mathcal{X}}} \Lambda /\left(p^{a_{i}}\right) \oplus \bigoplus_{i} \Lambda /\left(f_{i}\right) .
$$

Si de plus $\mathrm{r}_{\mathcal{X}}$ est aussi trivial, alors

$$
\mathcal{X} \hookrightarrow \Lambda^{\rho \mathcal{X}} \oplus \bigoplus_{i} \Lambda /\left(f_{i}\right)
$$

et ainsi $\mathcal{X}$ est sans $\mathbb{Z}_{p}$-torsion. D'après la proposition 2.5 , le pro-p-groupe $H$ est libre et par conséquent $\operatorname{cd}(G) \leq \operatorname{cd}(H)+\operatorname{cd}\left(\mathbb{Z}_{p}\right) \leq 2$.

Remarque. (i) Lorsque $H$ est de type fini et non trivial ou que $G^{a b}$ a de la torsion, la dimension cohomologique $\operatorname{cd}_{p}(G)$ de $G$ est exactement 2 .

(ii) Lorsque $H^{2}\left(G, \mathbb{Q}_{p} / \mathbb{Z}_{p}\right)$ est nul, il vient également : $\rho_{\mathcal{X}}=-\chi_{2}(G):=$ $1-d_{p} G^{a b}+d_{p} H^{2}\left(G, \mathbb{F}_{p}\right)$.

2.2.2. Modules d'Iwasawa et corps de nombres. Reprenons les notations de la section 1 . Soient K un corps de nombres, $S$ un ensemble de places de $\mathrm{K}$ au-dessus de $p, T$ un ensemble fini de places de $\mathrm{K}$ disjoint de $S$, et $\mathrm{K}_{\infty}$ une $\mathbb{Z}_{p}$-extension de $\mathrm{K}$ contenue dans $\mathrm{K}_{S}$.

Lemme 2.4. Soit $\mathrm{K}_{n}$ le sous corps de $\mathrm{K}_{\infty}$ de degré $p^{n}$ sur $\mathrm{K}$. Lorsque $\widetilde{T}=$ $T$ (c'est-à-dire les places de $T$ sont totalement décomposées dans $\mathrm{K}_{\infty} / \mathrm{K}$ ), il vient

$$
\operatorname{Gal}\left(\mathrm{K}_{n, S}^{T, a b} / \mathrm{K}_{\infty}\right) \simeq \mathcal{X}_{S}^{T} / \omega_{n} \mathcal{X}_{S}^{T}
$$


Preuve. Contentons-nous de montrer le lemme pour $T=\emptyset$. Soit $\mathrm{K}_{n, S}^{a b}$ l'extension abélienne maximale de $\mathrm{K}_{n}$ contenue dans $\mathrm{K}_{S}$. C'est aussi la pro- $p$-extension abélienne maximale de $\mathrm{K}_{n}$ non-ramifiée en dehors de $S$. Soit $F_{n}$ le corps maximal fixé par $\omega_{n} \mathcal{X}=\left((1+X)^{p^{n}}-1\right) \mathcal{X}$.

Le groupe de Galois $\operatorname{Gal}\left(\mathrm{K}_{\infty} / \mathrm{K}_{n}\right)=<\gamma^{p^{n}}>$ agit trivialement sur le quotient $\mathcal{X} / \omega_{n} \mathcal{X}$ (se rappeler : $\gamma \leftrightarrow X+1$ ). Ainsi, $\mathrm{Gal}\left(\mathrm{K}_{\infty} / \mathrm{K}_{n}\right)$ étant cyclique, l'extension $F_{n} / \mathrm{K}_{n}$ est abélienne. Par maximalité de $\mathrm{K}_{n, S}^{a b}$, on a $F_{n} \subset \mathrm{K}_{n, S}^{a b}$. L'inclusion inverse est évidente $: \mathrm{K}_{n, S}^{a b} / \mathrm{K}_{n}$ étant abélienne, $\operatorname{Gal}\left(\mathrm{K}_{\infty} / \mathrm{K}_{n}\right)$ agit trivialement sur $\operatorname{Gal}\left(\mathrm{K}_{n, S}^{a b} / \mathrm{K}_{\infty}\right)$. Ainsi $\operatorname{Gal}\left(\mathrm{K}_{\infty} / \mathrm{K}_{n}\right)$ est un quotient de $\mathcal{X} / \omega_{n}$ et $\mathrm{K}_{n, S}^{a b} \subset F_{n}$.

Soit $\mathrm{K}_{n_{0}}$ un corps de nombres de $\mathrm{K}_{\infty} / \mathrm{K}$ où toutes les places ramifiées et inertes de $S$ et $T$ dans l'extension $\mathrm{K}_{\infty} / \mathrm{K}$ le sont totalement dans $\mathrm{K}_{\infty} / \mathrm{K}_{n_{0}}$.

Lemme 2.5. (i) Il existe un sous- $\Lambda$-module $\mathcal{Y}$ de $\mathcal{X}_{T}^{S}$ tel que pour $n \geq n_{0}$,

$$
\operatorname{Gal}\left(\mathrm{K}_{n, T}^{S, a b} / \mathrm{K}_{n}\right) \simeq \mathcal{X}_{T}^{S} / \omega_{n, n_{0}} \mathcal{Y}
$$

(ii) Lorsque $T$ est différent de $\tilde{T}$, il existe un sous- $\Lambda$-module $\mathcal{Y}$ de $\mathcal{X}_{S}^{T}$ tel que pour $n \geq n_{0}$,

$$
\operatorname{Gal}\left(\mathrm{K}_{n, S}^{T, a b} / \mathrm{K}_{n}\right) \simeq \mathcal{X}_{S}^{T} / \omega_{n, n_{0}} \mathcal{Y}
$$

Preuve. Il suffit de reprendre la preuve du lemme 13.18 de [31] tout en notant que : (i) toute extension $\mathrm{K}^{\prime} / \mathrm{K}$ de $\mathrm{L}_{T}^{S} / \mathrm{K}, S \mid \widetilde{S}$-décomposée, est $S$-décomposée ; (ii) toute extension $\mathrm{K}^{\prime} / \mathrm{K}$ de $\mathrm{L}_{S}^{T} / \mathrm{K}, T \mid \widetilde{T}$-décomposée, est $T$-décomposée.

Remarque. Le lemme de Nakayama indique que les $\Lambda$-modules $\mathcal{X}_{T}^{S}$ et $\mathcal{X}_{S}^{T}$ sont de type fini.

Proposition 2.8. Notons par $G_{n, S}$ (reps. $G_{n, T}^{S}$ ) le groupe de Galois $\operatorname{Gal}\left(\mathrm{K}_{n, S} / \mathrm{K}_{n}\right)$ (resp. $\left.\operatorname{Gal}\left(\mathrm{K}_{n, T}^{S} / \mathrm{K}_{n}\right)\right)$.

(i) S'il existe deux entiers $m>n \geq 0$ pour lesquels $d_{p} G_{m, S}=d_{p} G_{n, S}$, alors $\rho_{S}=\mathrm{r}_{S}=0$

(ii) S'il existe deux entiers $m>n \geq n_{0}$ pour lesquels $d_{p} G_{n, T}^{S}=d_{p} G_{m, T}^{S}$, alors $\rho_{T}^{S}=\mathrm{r}_{T}^{S}=0$.

Preuve. Ces deux points sont une conséquence immédiate des lemmes 2.2, 2.4 et 2.5 .

\section{Les conditions de type "Leopoldt"}

\subsection{Quelques évidences.}

Définition et Proposition. Les deux assertions suivantes sont équivalentes 
(1) $\mathbb{Q}_{p} \otimes_{\mathbb{Z}_{p}} G_{S}^{a b}$ est un $\mathbb{Q}_{p}$-espace vectoriel de dimension $\delta_{S}-\left(r_{1}+r_{2}-1\right)$

(2) Le morphisme de $\mathbb{Z}_{p}$-modules $\varphi_{S}: \mathbb{Z}_{p} \otimes_{\mathbb{Z}_{p}} E_{K} \rightarrow \prod_{\mathfrak{p} \in S} U_{\mathfrak{p}}^{1}$ est injectif où $\delta_{S}=\sum_{\mathfrak{p} \in S}\left[\mathrm{~K}_{\mathfrak{p}}: \mathbb{Q}_{p}\right]$. Lorsque ces conditions sont remplies, nous disons que $\mathrm{K}$ vérifie la $S$-condition arithmétique.

Définition. Lorsque le groupe $H^{2}\left(G_{S}, \mathbb{Q}_{p} / \mathbb{Z}_{p}\right)$ est trivial (ou encore quand $\left.H_{2}\left(G_{S}, \mathbb{Z}_{p}\right)=0\right)$, nous disons que $\mathrm{K}$ vérifie la $S$-condition cohomologique.

Lorsque $S$ contient l'ensemble des places au-dessus de $p$, il y a équivalence entre la $S$-condition cohomologique et la $S$-condition arithmétique (cf. par exemple [24]). Pour le cas général, nous avons besoin du lemme suivant (par exemple, cf. [20], [7] appendice) :

\section{Lemme 3.1.}

$$
d_{p} H_{2}\left(G_{S}, \mathbb{Z}_{p}\right) \leq \mathrm{rk}_{\mathbb{Z}_{p}} E_{\mathrm{K}}-\operatorname{rk}_{\mathbb{Z}_{p}} \operatorname{Im}\left(\varphi_{S}\right)
$$

C'est plus ou moins une conséquence du lemme 3.2 à suivre.

Proposition 3.1. La S-condition arithmétique implique la $S$-condition cohomologique.

Preuve. Si la $S$-condition arithmétique est vérifiée, alors $d_{p} H_{2}\left(G_{S}, \mathbb{Z}_{p}\right)=$ 0 . Le lemme de Nakayama appliqué au $\mathbb{Z}_{p}$-module compact $H_{2}\left(G_{S}, \mathbb{Z}_{p}\right)$ permet de conclure.

Notons que, comme pour la conjecture de Leopoldt, nous pouvons avancer deux $S$-conditions faibles.

Définition. Soient $\mathrm{K}_{\infty} / \mathrm{K}$ une $\mathbb{Z}_{p}$-extension de $\mathrm{K}$ contenue dans $\mathrm{K}_{S}$ et $H_{S}=\operatorname{Gal}\left(\mathrm{K}_{S} / \mathrm{K}_{\infty}\right)$. Nous disons que le couple $(\mathrm{K}, S)$ vérifie

- la $S$-condition arithmétique faible de Leopoldt pour $\mathrm{K}_{\infty} / \mathrm{K}$, si le $p$-rang du noyau de $\varphi_{n, S}: \mathbb{Z}_{p} \otimes_{\mathbb{Z}} E_{\mathrm{K}_{n}} \rightarrow U_{S_{n}}$ est borné ;

- la $S$-condition cohomologique faible de Leopoldt pour $\mathrm{K}_{\infty} / \mathrm{K}$, si le groupe $H_{2}\left(H_{S}, \mathbb{Z}_{p}\right)$ est trivial.

Proposition 3.2. 1) La S-condition cohomologique forte entraine la $S$-condition cohomologique faible (pour toutes les $\mathbb{Z}_{p}$-extensions $\mathrm{K}_{\infty} / \mathrm{K}$, contenue dans $\left.\mathrm{K}_{S} / \mathrm{K}\right)$.

2) La S-condition arithmétique (resp. cohomologique) faible pour $\mathrm{K}_{\infty} / \mathrm{K}$ implique que le p-rang des groupes $H_{2}\left(G_{n, S}, \mathbb{Z}_{p}\right)$ est borné $\left(\bigcup \mathrm{K}_{n}=\mathrm{K}_{\infty}\right)$.

3) $S i \operatorname{cd}_{p}\left(G_{S}\right) \leq 2$, la $S$-condition arithmétique faible entraine la $S$-condition cohomologique faible.

4) La S-condition arithmétique faible équivaut à $\rho_{S}=\delta_{S}-\left(r_{1}+r_{2}\right)$. 
Preuve. 1) La $S$-condition cohomologique se traduit par la trivialité du groupe $H^{2}\left(G_{S} / \mathbb{Q}_{p} / \mathbb{Z}_{p}\right)$. Maintenant la suite exacte de la proposition 2.6 montre que $H^{2}\left(H_{S}, \mathbb{Q}_{p} / \mathbb{Z}_{p}\right)^{\Gamma}$ est trivial. Il en est de même pour $H^{2}\left(H_{S}, \mathbb{Q}_{p} / \mathbb{Z}_{p}\right)$.

2) La condition $H_{2}\left(H_{S}, \mathbb{Z}_{p}\right)=0$ implique $H_{2}\left(G_{n, S}, \mathbb{Z}_{p}\right) \simeq \mathcal{X}_{S}^{\omega_{n}}$. Ainsi, la suite des $p$-rang des groupes $H_{2}\left(G_{n, S}, \mathbb{Z}_{p}\right)$ est bornée.

Enfin, le lemme 3.1 indique que si le $S$-défaut de Leopoldt est borné dans $\mathrm{K}_{\infty} / \mathrm{K}$ par $m$, alors le $p$-rang de $H_{2}\left(G_{n, S}, \mathbb{Z}_{p}\right)$ est aussi borné par $m$.

3) On s'inspire de [23], chapitre V, lemme 5.6.13. Puisque $G_{S}$ est de dimension cohomologique au plus 2 , le groupe $H_{2}\left(H_{S}, \mathbb{Z}_{p}\right)$ est un $\Lambda$-module libre. Si la $S$-condition cohomologique faible est vérifiée, d'après le point 2) le $p$-rang des groupes $H_{2}\left(G_{n, S}, \mathbb{Z}_{p}\right)$ est borné. De $\underset{\hbar}{\lim } H_{2}\left(G_{n, S}, \mathbb{Z}_{p}\right)=$ $H_{2}\left(H_{S}, \mathbb{Z}_{p}\right)$, on conclut alors aisément.

4) On rappelle que pour tout $n$,

$$
\operatorname{rk}_{\mathbb{Z}_{p}} G_{n, S}^{a b}=p^{n} \delta_{S}-\operatorname{rk}_{\mathbb{Z}_{p}} \operatorname{Im}\left(\varphi_{S_{n}}\right)=p^{n}\left(\delta_{S}-\left(r_{1}+r_{2}\right)\right)+1+\operatorname{rk}_{\mathbb{Z}_{p}} \operatorname{ker}\left(\varphi_{S_{n}}\right)
$$

Mais, d'un autre coté, le théorème de structure nous indique que $\operatorname{rk}_{\mathbb{Z}_{p}} G_{n, S}^{a b}=$ $p^{n} \rho_{S}+O(1)$. On conclut alors facilement.

3.2. $S$-conditions et dimension cohomologique. Commencons par remarquer que la dimension cohomolgique de $G_{S}$ est fortement liée à la $S$-condition arithmétique.

Proposition 3.3. (i) Si la S-condition arithmétique faible est vérifiée le long de $\mathrm{K}_{S} / \mathrm{K}$, c'est à dire s'il existe une constante c telle que pour tout corps de nombres $\mathrm{L}$ contenu dans $\mathrm{K}_{S} / \mathrm{K}$, le $\mathbb{Z}_{p}$-rang du noyau de $\varphi_{\mathrm{L}, S}:=$ $E_{\mathrm{L}} \rightarrow U_{\mathrm{L}, S}^{1}$ est borné par c, alors ou bien $G_{S}$ est fini ou bien la dimension cohomologique de $G_{S}$ est au plus 2 .

(ii) Si la S-dimension arithmétique forte est vérifiée le long de $\mathrm{K}_{S} / \mathrm{K}$, alors la dimension cohomologique de $G_{S}$ est au plus 2 .

Avant de passer à la preuve de ceci, nous rappellerons un lemme bien connu.

Lemme 3.2. Soit $G$ un pro-p-groupe pour lequel $H^{1}\left(G, \mathbb{F}_{p}\right)$ et $H^{2}\left(G, \mathbb{F}_{p}\right)$ sont finis. Alors

$$
d_{p} H_{2}\left(G, \mathbb{Z}_{p}\right)-\operatorname{rk}_{\mathbb{Z}_{p}} G^{a b}=\chi_{2}(G)-1,
$$

où $\chi_{2}(G)$ est la caractéristique d'Euler-Poincaré de $G$ tronquée à l'ordre 2.

Preuve. La preuve de la proposition 2.5 a fait apparaitre l'identité $d_{p} H^{2}\left(G, \mathbb{F}_{p}\right)=d_{p} H_{2}\left(G, \mathbb{Z}_{p}\right)+d_{p} G^{a b}[p]$.

On a donc bien : $d_{p} H^{2}\left(G, \mathbb{F}_{p}\right)=d_{p} H_{2}\left(G, \mathbb{F}_{p}\right)=d_{p} H_{2}\left(G, \mathbb{Z}_{p}\right)+d_{p} G^{a b}-$ $\mathrm{rk}_{\mathbb{Z}_{p}} G^{a b}$. 
Remarque. C'est en partie ce lemme qui permet de montrer le lemme 3.1.

Preuve de la proposition 3.3. Avant toute chose, rappelons que pour un pro$p$-groupe $M$, la caractéristique d'Euler-Poincaré $\chi_{2}(M)$ tronquée à l'ordre 2 est définie par : $\chi_{2}(M):=1-d_{p} H^{1}\left(M, \mathbb{F}_{p}\right)+d_{p} H^{2}\left(M, \mathbb{F}_{p}\right)$, lorsque tout ceci a un sens.

(i) Soit $U$ un sous-groupe ouvert de $G_{S}$. Notons par L le corps de nombres associé par la théorie de Galois à $U$. D'après le lemme 3.2, $\chi_{2}(U)=1-$ $\mathrm{rk}_{\mathbb{Z}_{p}} U^{a b}+d_{p} H_{2}\left(U, \mathbb{Z}_{p}\right)=1-(G: U) \delta_{S}+\operatorname{rk}_{\mathbb{Z}_{p}} \operatorname{Im} \varphi_{\mathrm{L}, S}+d_{p} H_{2}\left(U, \mathbb{Z}_{p}\right)$ et également $\chi_{2}\left(G_{S}\right)=1-\delta_{S}+\mathrm{rk}_{\mathbb{Z}_{p}} \operatorname{Im} \varphi_{S}+d_{p} H_{2}\left(G, \mathbb{Z}_{p}\right)$.

Il vient ainsi, d'après le lemme $3.1, \chi_{2}\left(G_{S}\right) \leq r_{1}+r_{2}-\delta_{S}$.

Si l'on suppose que dans l'extension $\mathrm{K}_{S} / \mathrm{K}$ le défaut de la $S$-condition arithmétique est borné par $c$, alors $\mathrm{rk}_{\mathbb{Z}_{p}} U^{a b} \leq(\mathrm{L}: \mathrm{K})\left(\delta_{S}-\left(r_{1}+r_{2}\right)\right)+c+1$. Il vient ainsi $\chi_{2}(U) \geq d_{p} H_{2}\left(U, \mathbb{Z}_{p}\right)-(\mathrm{L}: \mathrm{K})\left(\delta_{S}-\left(r_{1}+r_{2}\right)\right)-c$. Par conséquent, $c+\chi_{2}(U) \geq\left[G_{S}: U\right]\left(r_{1}+r_{2}-\delta_{S}\right) \geq\left[G_{S}: U\right] \chi_{2}\left(G_{S}\right)$. On conclut avec le résultat de Schmidt (cf. proposition 2.2).

(ii) Supposons la $S$-condition arithmétique satisfaite le long de $\mathrm{K}_{S} / \mathrm{K}$. Pour tout ouvert $U$ de $G_{S}$, le groupe $H_{2}\left(U, \mathbb{Z}_{p}\right)$ est trivial. Il vient ainsi : $\chi_{2}(U)=\left(G_{S}: U\right)\left(\delta_{S}-\left(r_{1}+r_{2}\right)\right)$. Par conséquent, $\chi_{2}$ est multiplicatif pour les ouverts de $G_{S}$. Le point 3 de la proposition 2.1 permet de conclure.

Nous venons de voir qu'une certaine condition arithmétique le long de $G_{S}$ impliquait que la dimension de $G_{S}$ est au plus 2. Citons un autre résultat dans ce sens.

Proposition 3.4. Si $\mathrm{K}$ vérifie la $S$-condition arithmétique et s'il existe une $\mathbb{Z}_{p}$-extension $\mathrm{K}_{\infty} / \mathrm{K}$ pour laquelle $\mathrm{r}_{S}=0$, alors $\operatorname{cd}_{p}\left(G_{S}\right) \leq 2$.

Preuve. D'après la proposition $3.1, H^{2}\left(G_{S}, \mathbb{Q}_{p} / \mathbb{Z}_{p}\right)=0$. La conclusion découle alors immédiatement de la proposition 2.7 .

C'est ce résultat que nous utiliserons. Ces conditions sont parfaitement adaptées aux calculs : la condition arithmétique se teste après réduction modulo une puissance $p^{i}$ convenable ; la condition $\mathrm{r}_{S}=0$ se teste avec le lemme de Nakayama (après quelques calculs dans les premiers étages de $\mathrm{K}_{\infty} / \mathrm{K}$ : voir la proposition 2.8 pour une première version brute, et la section 5 pour une version plus fine).

Notons que le cas des corps quadratiques trouve une réponse immédiate grâce à un résultat de Gillard [6].

Proposition 3.5. Soit $\mathrm{K} / \mathrm{K}_{0}$ une extension abélienne d'un corps quadratique imaginaire $\mathrm{K}_{0}$. Soit $S \subset S_{p}$ un sous-ensemble non-vide de places de $\mathrm{K}$ au-dessus de $p$ stable par $\mathrm{Gal}\left(\mathrm{K} / \mathrm{K}_{0}\right)$. Enfin, soit $G_{S}=\mathrm{Gal}\left(\mathrm{K}_{S} / \mathrm{K}\right)$.

Alors la dimension cohomologique de $G_{S}$ est au plus 2. 
Preuve. Si $S$ contient toutes les places au-dessus de $p$, c'est bien connu. Supposons que $S$ ne contient qu'une place au-dessus de $p$. L'extension $\mathrm{K}_{0, S} / \mathrm{K}_{0}$ ne contient qu'une $\mathbb{Z}_{p}$-extension $\mathrm{K}_{0, \infty}$. Gillard dans [6] a montré que pour la $\mathbb{Z}_{p}$-extension $\mathrm{K}_{0, \infty} \mathrm{K} / \mathrm{K}$, l'invariant $\mu_{S}$ de $\operatorname{Gal}\left(\mathrm{K}_{S} / \mathrm{K}_{0, \infty} \mathrm{K}\right)^{a b}$ est trivial (cf. également [28]).

Ensuite $\varphi_{S}$ est injective, c'est classique. Le théorème 3.4 s'applique.

Remarque. (i) Plus généralement, Wingberg, dans [32], montre que si $\rho_{S}=0$ et que si la $S$-condition arithmétique faible est vérifiée, alors $\mathcal{X}_{S}$ n'a pas de sous-module fini. Si l'on suppose de plus $\mathrm{r}_{S}=0$, on obtient alors que le groupe $\mathrm{Gal}\left(\mathrm{K}_{S} / \mathrm{K}_{\infty}\right)$ est libre.

(ii) On peut noter que la conjecture de Schanuel (cf. [14], [20], ...) associée à la proposition 3.4 permet de remplacer dans la proposition 3.5 la condition " $\mathrm{K} / \mathrm{K}_{0}$ abélienne" par " $\mathrm{K} / \mathrm{K}_{0}$ galoisienne".

Enfin, nous avons vu que la condition arithmétique impliquait la condition cohomologique. Que peut-on dire de la réciproque?

Proposition 3.6. Soient $\mathrm{K}$ un corps de nombres et $S \subset S_{p}$ un sousensemble de places de $\mathrm{K}$ au-dessus de $p$. Soit $\mathrm{K}_{S}$ la pro-p-extension maximale de $\mathrm{K}, S$-ramifiée ; $G_{S}=\mathrm{Gal}\left(\mathrm{K}_{S} / \mathrm{K}\right)$. Supposons $G_{S}$ de dimension cohomologique au plus 2. Alors pour toute extension $\mathrm{L} / \mathrm{K}$ de corps de nombres contenue dans $\mathrm{K}_{S} / \mathrm{K}$, il vient :

$$
d_{p} H_{2}\left(G_{\mathrm{L}, S}, \mathbb{Z}_{p}\right)-d_{p} \operatorname{ker}\left(\varphi_{\mathrm{L}, S}\right)=[\mathrm{L}: \mathrm{K}]\left(d_{p} H_{2}\left(G_{\mathrm{K}, S}, \mathbb{Z}_{p}\right)-d_{p} \operatorname{ker}\left(\varphi_{\mathrm{K}, S}\right)\right)
$$

Preuve. Soit $H=\mathrm{Gal}\left(\mathrm{K}_{S} / \mathrm{L}\right)$. Comme $G_{S}$ est de dimension cohomologique au plus 2, la caractéristique d'Euler-Poincaré s'arrête au plus au troisième terme et elle est multiplicative dans $\mathrm{K}_{S} / \mathrm{K}$. Il suffit alors de noter que $\mathrm{rk}_{\mathbb{Z}_{p}} G_{\mathrm{L}, S}=r k_{\mathbb{Z}_{p}} H^{a b}=\delta_{\mathrm{L}, S}-[\mathrm{L}: \mathrm{K}]\left(r_{1}+r_{2}\right)+1+\operatorname{ker}\left(\varphi_{\mathrm{L}}, S\right)$.

On en déduit immédiatement le corollaire suivant :

Corollaire 3.1. (i) $S i \mathrm{~cd}_{p}\left(G_{S}\right)=1$, la $S$-condition arithmétique est vérifiée pour $(\mathrm{K}, S)$ si et seulement si elle est conservée dans $\mathrm{K}_{S} / \mathrm{K}$.

(ii) Supposons que la dimension cohomologique de $G_{S}$ est au plus 2 et que $\mathrm{K}$ vérifie la $S$-condition arithmétique. Alors, pour toute extension finie $\mathrm{L} / \mathrm{K}$ contenue dans $\mathrm{K}_{S}$,

$$
d_{p} \operatorname{ker}\left(\varphi_{\mathrm{L}, S}\right)=d_{p} H_{2}\left(G_{\mathrm{L}, S}, \mathbb{Z}_{p}\right)
$$

En particulier, dans ce cas, il y a équivalence entre la $S$-condition cohomologique et la $S$-condition arithmétique.

3.3. $S$-conditions et polynôme caractéristique. Des informations sur les $S$-conditions cohomologiques peuvent se déduire à partir du $\Lambda$-module $\mathcal{X}_{S}$, le point de départ étant la proposition suivante : 
Proposition 3.7. Soit $\mathrm{K}_{\infty} / \mathrm{K}$ une $\mathbb{Z}_{p}$-extension contenue dans $\mathrm{K}_{S} / \mathrm{K}$. Alors la $S$-condition cohomologique est vérifiée pour $\mathrm{K}$ si et seulement si la $S$-condition cohomologique faible l'est et $\mathcal{X}_{S}{ }^{X}=\left(\mathcal{X}_{S}\right)^{\Gamma}=0$.

Preuve. C'est la proposition 2.6 .

Proposition 3.8. Supposons que $\mathcal{X}_{S}$ n'a pas de sous $\Lambda$-module fini. Alors, la $S$-condition cohomologique est vraie à l'étage $\mathrm{K}_{n}$ de $\mathrm{K}_{\infty} / \mathrm{K}$ si et seulement si, la $S$-condition cohomologique faible l'est et si le polynôme caractéristique $P_{S}$ de $\mathcal{X}_{S}$ est premier avec $\omega_{n}$.

La preuve de cette proposition repose sur le lemme suivant :

Lemme 3.3. Soit $\mathcal{X}$ un $\Lambda$-module de type fini n'ayant pas de sous- $\Lambda$-module fini. Alors, pour tout $n, \mathcal{X}^{\omega_{n}}=0$ équivaut $\grave{a} \omega_{n}$ et $P_{\mathcal{X}}$ premiers entre eux, où $P_{\mathcal{X}}=\prod_{i} f_{i}^{b_{i}}$ est le polynôme caractéristique de $\mathcal{X}$.

Preuve. Par hypothèse, il existe un morphisme injectif de $\Lambda$-modules $\phi$ : $\mathcal{X} \rightarrow \mathcal{E}=\Lambda^{\rho} \oplus \bigoplus_{i} \Lambda /\left(f_{i}^{b_{i}}\right)$ à conoyau fini.

Soit $x \in \mathcal{X}$ tel que $\omega_{n} x=0$. Alors $\omega_{n} \phi(x)=0$. Si pour tout $i,\left(f_{i}, \omega_{n}\right)=$ 1 , alors $\phi(x)=0$ et ainsi $x=0$.

Réciproquement. Supposons $\omega_{n}$ et $f_{i}$ non premiers entre eux, pour un certain indice $i$. Il existe $y \in \mathcal{E}$ avec $\mathbb{Z}_{p} y \simeq \mathbb{Z}_{p}$ et tel que $\omega_{n} y=0$. D'autre part, il existe également $b \in B$ et $x \in \mathcal{X}$ tels que $y=\phi(x)+b$. En

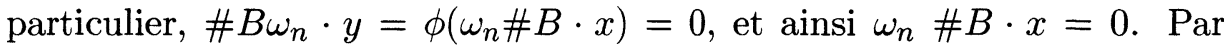
conséquent, $\# B \cdot x \in \mathcal{X}^{\omega_{n}}$. Comme $\left\langle y>_{\mathbb{Z}_{p}}\right.$ est sans-torsion, on en déduit que $\mathcal{X}^{\omega_{n}}$ est non trivial.

Preuve de la proposition 3.8. Supposons la $S$-condition cohomologique vérifiée pour $\mathrm{K}_{n}$. Alors, d'une part, la $S$-condition cohomologique faible est satisfaite : $H_{2}\left(H_{S}, \mathbb{Z}_{p}\right)$ est trivial. Et d'autre part, d'après la proposition 2.6 et le lemme $3.3, P_{S}$ et $\omega_{n}$ sont premiers entre eux. La conclusion en découle.

La réciproque se montre de façon identique.

Corollaire 3.2. (i) Si la S-condition arithmétique forte est satisfaite pour un étage $\mathrm{K}_{n}$ de $\mathrm{K}_{\infty} / \mathrm{K}$, alors la $S$-condition arithmétique faible est satisfaite pour $\mathrm{K}_{\infty} / \mathrm{K}$.

(ii) Supposons la $S$-condition arithmétique faible vérifiée pour $\mathrm{K}_{\infty} / \mathrm{K}$. Alors pour tout étage $\mathrm{K}_{n}$ de $\mathrm{K}_{\infty} / \mathrm{K}$, la $S$-condition arithmétique forte et la $S$-condition cohomologique forte sont équivalentes.

Preuve. (i) Supposons la $S$-condition arithmétique forte réalisée pour $\mathrm{K}_{n}$. La $S$-condition cohomologique forte est donc aussi réalisée pour le corps $\mathrm{K}_{n}$. Alors, $\mathcal{X}_{S}$ n'a pas de sous- $\mathbb{Z}_{p}\left[\left[\Gamma^{p^{n}}\right]\right]$-module fini et donc n'a pas de 
sous $\Lambda$-module fini. D'autre part, d'après la proposition 3.8 , le polynôme $P_{S}=\prod_{i} f_{i}^{b_{i}}$ est premier à $\omega_{n}$. Maintenant, il est bien connu (cf. [31], par exemple), que pour un polynôme irréductible $f, f$ et $\omega_{n}$ sont premiers entre eux si et seulement si, le quotient $\Lambda /\left(f, \omega_{n}\right)$ est fini. Ainsi, $\mathrm{rk}_{\mathbb{Z}_{p}} \mathcal{X}_{S} / \omega_{n} \mathcal{X}_{S}=$ $p^{n} \rho_{S}+\sum_{i} \operatorname{rk}_{\mathbb{Z}_{p}} \Lambda /\left(f_{i}^{b_{i}}, \omega_{n}\right)=p^{n} \rho_{S}$. Il suffit ensuite de se rappeler que $\operatorname{rk}_{\mathbb{Z}_{p}} \mathcal{X}_{S} / \omega_{n} \mathcal{X}_{S}=\operatorname{rk}_{\mathbb{Z}_{p}} G_{n, S}^{a b}-1=p^{n}\left(\delta_{S}-\left(r_{1}+r_{2}\right)\right)+\operatorname{rk}_{\mathbb{Z}_{p}} \operatorname{ker}\left(\varphi_{S_{n}}\right)=p^{n}\left(\delta_{S}-\right.$ $\left.\left(r_{1}+r_{2}\right)\right)$, ceci par hypothèse. Ainsi, $\rho_{S}=\delta_{S}-\left(r_{1}+r_{2}\right)$, ce qui indique que la $S$-condition arithmétique faible est vérifiée dans $\mathrm{K}_{\infty} / \mathrm{K}$.

(ii) Il faut donc montrer, pour un étage $\mathrm{K}_{n}$ de $\mathrm{K}_{\infty} / \mathrm{K}$, que la $S$-condition cohomologique implique la $S$-condition arithmétique.

Supposons donc satisfaite la $S$-condition cohomologique à l'étage $\mathrm{K}_{n}$. Alors $\mathcal{X}_{S}$ n'a pas de sous- $\Lambda$-module fini. La proposition 3.8 nous indique que $P_{S}$ est premier à $\omega_{n}$. Par conséquent, $\mathrm{rk}_{\mathbb{Z}_{p}} G_{n, S}^{a b}-1=\mathrm{rk}_{\mathbb{Z}_{p}} \mathcal{X}_{S} / \omega_{n} \mathcal{X}_{S}=p^{n} \rho_{S}$. Mais par hypothèse, $\rho_{S}=\delta_{S}-\left(r_{1}+r_{2}\right)$. Et ainsi, $\varphi_{S_{n}}: \mathbb{Z}_{p} \otimes E_{\mathrm{K}_{n}} \rightarrow U_{S_{n}}^{1}$ est injectif.

Pour terminer, notons qu'un nombre fini de calculs le long de $\mathrm{K}_{\infty} / \mathrm{K}$ permet de s'assurer dans certains cas si la $S$-condition arithmétique forte le long de $K_{\infty} / \mathrm{K}$ est réalisée ou non. En effet :

Corollaire 3.3. Supposons la quantité $\mathrm{rk}_{\mathbb{Z}_{p}} G_{n, S}^{a b}-p^{n} \rho_{S}$ bornée par une constante $c+1$. Alors la $S$-condition arithmétique est vérifiée pour tous les étages $\mathrm{K}_{n}$ de $\mathrm{K}_{\infty} / \mathrm{K}$ si et seulement si, elle est vérifiée pour le corps $\mathrm{K}$ ainsi que le corps $\mathrm{K}_{m}$ où $m$ est le plus grand entier vérifiant $(p-1) p^{m-1} \leq c$.

Preuve. Supposons assurée la $S$-condition arithmétique pour K. Ainsi : (i) la $S$-condition arithmétique faible est satisfaite dans $\mathrm{K}_{\infty} / \mathrm{K}$ (cf. corollaire 3.2); (ii) $H_{2}\left(G_{S}, \mathbb{Z}_{p}\right)$ est trivial.

Soit $m$ le plus grand entier vérifiant $(p-1) p^{m} \leq c$.

D'après la proposition 3.8 , pour $n \leq m, P_{S}$ et $\omega_{n}$ sont premiers entre eux. Pour conclure, il suffit alors de noter que pour $j>0$, nécessairement $f_{i}$ et $\omega_{m+j}$ sont aussi premiers entre eux : en effet, sinon, $\left(\omega_{m+j, m}\right)=\left(f_{i}\right)$ pour un certain entier $j>0$ (se rappeler que les polynômes $f_{i}$ sont irréductibles) et alors, on aurait $\sum_{i} \Lambda /\left(f_{i}, \omega_{m+j}\right)>c$, et ainsi, $\mathrm{rk}_{\mathbb{Z}_{p}} G_{m+j, S}-p^{m+j} \rho_{S}=$ $\operatorname{rk}_{\mathbb{Z}_{p}} \mathcal{X}_{S} / \omega_{m+j}+1-p^{m+j} \rho_{S}=d_{p} \operatorname{ker}\left(\varphi_{S_{m+j}}\right)+\sum_{i} \Lambda /\left(f_{i}, \omega_{m+j}\right)+1>$ $c+1$, d'où la contradiction. La proposition 3.8 s'applique : la $S$-condition cohomologique est vérifiée le long de $\mathrm{K}_{\infty} / \mathrm{K}$. D'après le corollaire 3.2 , il en est de même pour la $S$-condition arithmétique.

3.4. $S$-conditions et $\Lambda$-torsion de $\mathcal{X}_{S}$. Notons par $\mathcal{T}_{S}$ le sous- $\Lambda$-module de torsion de $\mathcal{X}_{S}$.

Le quotient $\mathcal{X}_{S} / \mathcal{T}_{S}$ est un $\Lambda$-module de type fini sans torsion. En particulier, $\mathcal{X}_{S} / \mathcal{T}_{S}$ n'a pas de sous-module fini. Le théorème de structure nous 
indique alors l'existence d'un $\Lambda$-module fini $B$ tel que l'on ait la suite exacte de $\Lambda$-modules :

$$
1 \longrightarrow \mathcal{X}_{S} / \mathcal{T}_{S} \longrightarrow \Lambda^{\rho_{S}} \longrightarrow B \longrightarrow 1
$$

Appliquant $\omega_{n}$ à cette suite puis utilisant le lemme du serpent, on obtient :

$$
1 \longrightarrow B^{\omega_{n}} \longrightarrow \mathcal{X}_{S} /\left(\mathcal{T}_{S}+\omega_{n} \mathcal{X}_{S}\right) \longrightarrow \Lambda^{\rho_{S}} / \omega_{n} \Lambda^{\rho_{S}}=\mathbb{Z}_{p}^{p^{n} \rho_{S}} \longrightarrow B / \omega_{n} B \longrightarrow 1
$$

Ainsi, $B^{\omega_{n}}$ est exactement la partie de torsion du $\mathbb{Z}_{p}$-module de type fini $\mathcal{X}_{S} /\left(\mathcal{T}_{S}+\omega_{n} \mathcal{X}_{S}\right)$.

Ce dernier $\Lambda$-module apparait dans la suite exacte :

$$
1 \longrightarrow\left(\mathcal{T}_{S}+\omega_{n} \mathcal{X}_{S}\right) / \omega_{n} \mathcal{X}_{S} \longrightarrow \mathcal{X}_{S} / \omega_{n} \mathcal{X}_{S} \longrightarrow \mathcal{X}_{S} /\left(\mathcal{T}_{S}+\omega_{n} \mathcal{X}_{S}\right) \longrightarrow 1
$$

où $\left(\mathcal{T}_{S}+\omega_{n}\right) \mathcal{X}_{S} / \omega_{n} \mathcal{X}_{S}$ est naturellement isomorphe au module $\mathcal{T}_{S} / \omega_{n} \mathcal{T}_{S}$.

Lemme 3.4. Si la $S$-condition cohomologique est vérifiée pour tout étage $\mathrm{K}_{n}$ de $\mathrm{K}_{\infty} / \mathrm{K}$, alors $\mathcal{T}_{S} / \omega_{n} \mathcal{T}_{S}$ est fini.

Preuve. En effet, le $\Lambda$-module $\mathcal{T}_{S} / \omega_{n} \mathcal{T}_{S}$ est annulé à la fois par $\omega_{n}$ et à la fois par $p^{\alpha} P_{S}$, où $\alpha=\max _{i} a_{i}$ (cf. section 2.2). Par hypothèse, ces polynômes sont premiers entre eux, et ainsi $\mathcal{T}_{S} / \omega_{n} \mathcal{T}_{S}$ est fini.

À présent, supposons vérifiée la $S$-condition cohomologique forte à chaque étage $\mathrm{K}_{n}$ de $\mathrm{K}_{\infty} / \mathrm{K}$. La suite exacte précédente de $\mathbb{Z}_{p}$-modules devient dans ce cas :

$$
1 \longrightarrow \mathcal{T}_{S} / \omega_{n} \mathcal{T}_{S} \longrightarrow \operatorname{Tor}_{\mathbb{Z}_{p}}\left(\mathcal{X}_{S} / \omega_{n} \mathcal{X}_{S}\right) \longrightarrow B^{\omega_{n}} \longrightarrow 1
$$

Ainsi,

Corollaire 3.4. Lorsque la $S$-condition cohomologique forte est vérifiée le long de $\mathrm{K}_{\infty} / \mathrm{K}$, le quotient $\mathcal{T}_{S} / \omega_{n} \mathcal{T}_{S}$ s'injecte dans la torsion de $G_{n, S}^{a b}$.

Ceci va nous permettre de montrer que si le rang de $\operatorname{Tor}_{\mathbb{Z}_{p}}\left(G_{n, S}^{a b}\right)$ ne croit pas suffisament vite, alors $\mathrm{r}_{S}$ est trivial.

Proposition 3.9. Supposons la S-condition cohomologique vérifiée à chaque étage $\mathrm{K}_{n}$ de $\mathrm{K}_{\infty} / \mathrm{K}$.

(a) S'il existe $n \geq 0$ pour lequel

$$
d_{p} \operatorname{Tor}_{\mathbb{Z}_{p}}\left(G_{n, S}^{a b}\right) \leq n,
$$

alors : (i) $\mathcal{T}_{S} / p \mathcal{T}_{S}$ est fini ; (ii) $\mathrm{r}_{S}=0$.

(b) $S$ 'il existe $n \geq 0$ pour lequel

$$
\text { \# } \operatorname{Tor}_{\mathbb{Z}_{p}}\left(G_{n, S}^{a b}\right) \leq p^{n}
$$

alors : (i) $\mathcal{T}_{S}$ est trivial; (ii) $\lambda_{S}=\mathrm{r}_{S}=0$. 
Preuve. (a). Si $n=0$, alors $\mathcal{T}_{S} / X \mathcal{T}_{S}=0$. Par le lemme de Nakayama, on en déduit que $\mathcal{T}_{S}$ est trivial.

Pour $n>0$, on a la suite de quotients :

$$
\mathcal{T}_{S} /\left(\mathcal{T}_{S}+\omega_{n} \mathcal{T}_{S}\right) \rightarrow \mathcal{T}_{S} /\left(p \mathcal{T}_{S}+\omega_{n-1} \mathcal{T}_{S}\right) \rightarrow \cdots \rightarrow \mathcal{T}_{S} /\left(p \mathcal{T}_{S}+X \mathcal{T}_{S}\right)
$$

Comme $d_{p} \mathcal{T}_{S} / \omega_{n} \mathcal{T}_{S} \leq n$, on est assuré que : ou bien $\mathcal{T}_{S} / X \mathcal{T}_{S}$ est trivial ; ou bien que pour un certain entier $m, \mathcal{T}_{S} /\left(p \mathcal{T}_{S}+\omega_{m} \mathcal{T}_{S}\right) \simeq \mathcal{T}_{S} /\left(p \mathcal{T}_{S}+\omega_{m+1} \mathcal{T}_{S}\right)$. Dans le premier cas, on a $\mathcal{T}_{S}=0$. Dans le second, il vient $p \mathcal{T}_{S}+\omega_{m} \mathcal{T}_{S}=$ $p \mathcal{T}_{S}+\omega_{m+1, m} \omega_{m} \mathcal{T}_{S}$, et ainsi $\omega_{m} \mathcal{T}_{S}+p \mathcal{T}_{S} / p \mathcal{T}_{S}$ est trivial, et ce par le lemme de Nakayama. Par conséquent, $\omega_{m} \mathcal{T}_{S} \subset p \mathcal{T}_{S}$. Ainsi, le quotient $\mathcal{T}_{S} / p \mathcal{T}_{S}$, qui est isomorphe à $\mathcal{T}_{S} /\left(\omega_{m} \mathcal{T}_{S}+p \mathcal{T}_{S}\right)$, est fini, car $\mathcal{T}_{S} / \omega_{m} \mathcal{T}_{S} \hookrightarrow \operatorname{Tor}_{\mathbb{Z}_{p}}\left(G_{m, S}^{a b}\right)$ l'est, ce qui prouve (i). D'après le lemme de Nakayama, le $\mathbb{Z}_{p}$-module compact $\mathcal{T}_{S}$ (car $\mathcal{X}_{S}$ l'est) est donc de type fini, et ainsi $\mathrm{r}_{S}=0$.

(b) Le point (i) implique clairement (ii).

Montrons donc (i).

Si $n=0$, alors $\mathcal{T}_{S} / X \mathcal{T}_{S}=0$. Par le lemme de Nakayama, on en déduit que $\mathcal{T}_{S}$ est trivial.

Pour $n>0$, on a la suite de quotients :

$$
\mathcal{T}_{S} / \omega_{n} \mathcal{T}_{S} \rightarrow \mathcal{T}_{S} / \omega_{n-1} \rightarrow \cdots \rightarrow \mathcal{T}_{S} / X \mathcal{T}_{S}
$$

Comme $\# \mathcal{T}_{S} / \omega_{n} \mathcal{T}_{S} \leq p^{n}$, on est assuré que : ou bien $\mathcal{T}_{S} / X \mathcal{T}_{S}$ est trivial; ou bien que pour un certain entier $m, \mathcal{T}_{S} / \omega_{m} \mathcal{T}_{S} \simeq \mathcal{T} / \omega_{m+1} \mathcal{T}_{S}$. Dans le premier cas, on a $\mathcal{T}_{S}=0$. Dans le second, il vient $\omega_{m+1, m} \omega_{m} \mathcal{T}_{S}=\omega_{m} \mathcal{T}_{S}$, et ainsi $\omega_{m} \mathcal{T}_{S}=0$. Or,

$$
\mathcal{T}_{S} \simeq \mathcal{T}_{S} / \omega_{m} \mathcal{T}_{S} \hookrightarrow \operatorname{Tor}_{\mathbb{Z}_{p}}\left(G_{m, S}^{a b}\right)
$$

Par conséquent, $\mathcal{T}_{S}$ est fini. La $S$-condition cohomologique étant vérifiée, $\mathcal{X}_{S}$ n'a donc pas de sous- $\Lambda$-module fini. En conclusion, $\mathcal{T}_{S}=0$.

Remarque. Restons dans la situation où la $S$-condition cohomologique le long de $\mathrm{K}_{\infty} / \mathrm{K}$ est vérifiée.

Supposons que la norme de $\operatorname{Tor}_{\mathbb{Z}_{p}}\left(G_{n, S}^{a b}\right)$ vers $\operatorname{Tor}_{\mathbb{Z}_{p}}\left(G_{n+1, S}^{a b}\right)$ est un isomorphisme pour un entier $n$. Alors, le diagramme commutatif

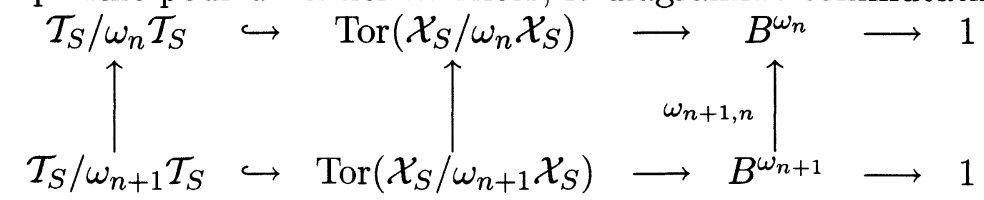

nous indique que $B^{\omega_{n+1}} \stackrel{\omega_{n+1, n}}{\longrightarrow} B^{\omega_{n}}$ est aussi un isomorphisme.

Le lemme du serpent appliqué à

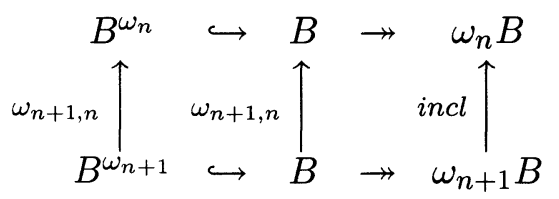


montre que $B^{\omega_{n+1, n}}$ est trivial. Par conséquent, d'après le lemme de Nakayama, $B$ l'est aussi. On obtient, dans ce cas, et pour tout $n$, que $G_{n, S}^{a b}$ est sans-torsion.

On peut alors faire la remarque suivante. Supposons les conditions de la proposition 3.9 satisfaites (cf. section 6). Pour $n>>0$, les groupes de torsion de $G_{n, S}^{a b}$ sont isomorphes, mais cet isomorphisme ne provient pas de la norme (lorsque ceux-ci ne sont pas triviaux). Ce qui indique que, pour $n>>0$, une partie de torsion à l'étage $n$ est absorbée par les $\mathbb{Z}_{p}$-extensions du $(n+1)$-ème étage. [14].

Pour plus de détails sur ce module de torsion, on renvoit par exemple à

\section{4. $\mathbb{Z}_{p}$-extensions et logarithme de Gras.}

Certains renseignements sur l'extension $\mathrm{K}_{S}^{a b} / \mathrm{K}$ s'obtiennent après calcul dans un corps de rayon $\mathrm{K}_{\mathfrak{m}}$ adapté. Par exemple, rappelons comment on peut calculer de cette façon le $p$-rang de $G_{S}$.

4.1. Calcul de $d_{p}\left(G_{S}\right)$. Le rayon à prendre est facile à déterminer lorsque $\mathrm{K}$ contient les racines $p$-èmes de l'unité. Il découle de la proposition suivante :

Proposition 4.1. Soit $K_{\mathfrak{p}} / \mathbb{Q}_{p}$ un corps local contenant les racines $p$-èmes de l'unité. Soit $\pi$ une uniformisante de $K_{\mathfrak{p}}$. Posons $\mathfrak{a}=p e\left(\mathrm{~K}_{\mathfrak{p}} / \mathbb{Q}_{p}\right) /(p-1)$. Le plus petit entier $n$ pour lequel $U^{n}$ est contenu dans $K_{\mathfrak{p}}^{p}$ est l'entier $\mathfrak{a}+1$. Ce dernier est appelé module d'hyperprimalité de $K_{\mathfrak{p}} / \mathbb{Q}_{p}$.

Corollaire 4.1. Supposons que les racines p-èmes de l'unité sont dans $\mathrm{K}$. Le compositum $\mathrm{L}$ des p-extensions élémentaires de $\mathrm{K}$ contenues dans $\mathrm{K}_{S}$ est contenu dans l'extension $\mathrm{K}_{\mathfrak{f}}$ de $\mathrm{K}$ de conducteur $\mathfrak{f}=\prod_{\mathfrak{p} \in S} \mathfrak{p}^{\mathfrak{a}_{\mathfrak{p}}+1}$, où $a_{\mathfrak{p}}=p e\left(\mathrm{~K}_{\mathfrak{p}} / \mathbb{Q}_{p}\right) /(p-1)$.

Corollaire 4.2. Soit le module $\mathfrak{m}=\prod_{\mathfrak{p} \in S} \mathfrak{p}^{\mathfrak{a}_{\mathfrak{p}}+1}$. Alors, lorsque $\mathrm{K}$ contient les racines $p$-èmes de l'unité, $d_{p} G_{S}=d_{p} \mathrm{Cl}_{\mathrm{K}, \mathfrak{m}}$.

4.2. Le logarithme de Gras. Fixons $S$ un ensemble fini de premiers de $\mathrm{K}$ au-dessus de $p$. Pour un idéal $\mathfrak{A}$ de $I_{\mathrm{K}, S}$ (idéaux fractionnaires de K premiers à $S$ ), le logarithme $\log (\mathfrak{A})$ de $\mathfrak{A}$ relativement à $S$ est défini comme suit : Soient $n \in \mathbb{N}$ et $x \in \mathcal{O}_{\mathrm{K}}$ tels que $\mathfrak{A}^{n}=(x)$. On pose $\log ^{\prime}(\mathfrak{A})=1 / n \prod_{\mathfrak{p} \in S} \log _{\mathfrak{p}}(x) \in \prod_{p} \mathrm{~K}_{\mathfrak{p}}$ puis $\log (\mathfrak{A})=\log ^{\prime}(\mathfrak{A})+$ $\mathbb{Q}_{p} \log ^{\prime}\left(E_{\mathrm{K}}\right) \bmod \mathbb{Q}_{p} \log ^{\prime}\left(E_{\mathrm{K}}\right)$.

On peut noter que le logarithme sur les idéaux de $I_{\mathrm{K}, S}$ est défini à partir d'un système de générateurs du groupes des classes de $\mathrm{K}$ et du logarithme 
$\log \left(P_{\mathrm{K}}\right)$ des idéaux principaux $P_{\mathrm{K}}$. Le théorème d'approximation indique alors que $\log \left(P_{\mathrm{K}}\right)=\prod_{\mathfrak{p} \in S} \log _{\mathfrak{p}}\left(U_{\mathfrak{p}}^{1}\right)+\mathbb{Q}_{p} \log ^{\prime}\left(E_{\mathrm{K}}\right) \bmod \mathbb{Q}_{p} \log ^{\prime}\left(E_{\mathrm{K}}\right)$.

Le théorème suivant est au coeur des calculs à venir. Il permet, entre autre, de décrire la loi de décomposition des idéaux premiers dans une $\mathbb{Z}_{p}$-extension.

Théorème 4.1 (Gras, cf. [7], chapitre III, théorème 2.5). 1) Notons par $\widetilde{\mathrm{K}_{S}}$ le compositum des $\mathbb{Z}_{p}$-extensions de $\mathrm{K}$ contenues dans $S$. Il vient alors la suite exacte :

$$
1 \longrightarrow \operatorname{Tor}\left(G_{S}^{a b}\right) \longrightarrow I_{\mathrm{K}, S} \stackrel{\log }{\longrightarrow}<\log \left(I_{\mathrm{K}, S}\right)>_{\mathbb{Z}_{p}} \longrightarrow 1,
$$

où $<\log \left(I_{\mathrm{K}, S}\right)>_{\mathbb{Z}_{p}} \stackrel{\operatorname{Artin}}{\simeq} \operatorname{Gal}\left(\widetilde{\mathrm{K}_{S}} / \mathrm{K}\right)$.

2) Pour $\mathfrak{p} \notin S$, le groupe de décomposition de $\mathfrak{p}$ dans $\widetilde{K_{S}} / K$ est donné par $\mathbb{Z}_{p} \log (\mathfrak{p})$.

3) L'ordre de la torsion $\operatorname{Tor}\left(G_{S}^{a b}\right)$ de $G_{S}^{a b}$ est donné par la formule :

$$
\begin{aligned}
& \left|\operatorname{Tor}\left(G_{S}^{a b}\right)\right|=\left|\operatorname{Tor}_{\mathbb{Z}_{p}}\left(U_{S}^{1} / \varphi_{\mathrm{K}, S}\left(E_{\mathrm{K}}\right)\right)\right| \times \frac{\left|\mathbb{Z}_{p} \otimes \mathrm{Cl}_{\mathrm{K}}\right|}{\left(\mathbb{Z}_{p} \log \left(\mathrm{Cl}_{\mathrm{K}}\right)+\log \left(U_{S}^{1}\right): \log \left(U_{S}^{1}\right)\right)} \\
& \text { 4) Enfin, }\left[\mathrm{K}^{H} \cap \widetilde{\mathrm{K}}_{S}: \mathrm{K}\right]=\left(\mathbb{Z}_{p} \log \left(\mathrm{Cl}_{\mathrm{K}}\right)+\log \left(U_{S}^{1}\right): \log \left(U_{S}^{1}\right)\right) \text {, où } \mathrm{K}^{H} \\
& \text { est le corps de Hilbert de } \mathrm{K} \text {. }
\end{aligned}
$$

À noter que dans [10], on trouve une généralisation du logarithme de Gras.

4.3. Recherche des $\mathbb{Z}_{p}$-extensions de $\mathrm{K}$. Commençons par une simple question. Soit $\mathrm{L}$ une sous-extension finie de $\mathrm{K}_{S} / \mathrm{K}$. A quelle condition, le corps de nombres L est-il un étage d'une $\mathbb{Z}_{p}$-extension de $\mathrm{K}$ ? C'est le "logarithme de Gras" qui va nous permettre de répondre explicitement à cette question. Précisons notre situation.

Soit un corps de nombres $\mathrm{K}$ contenant les racines $p$-èmes de l'unité. Soit $S \subset S_{p}$ un sous-ensemble de places de K tel que le $\mathbb{Z}_{p}$-rang de $G_{S}^{a b}$ est au moins 1 . Soit $\mathrm{K}_{i}$ le $i$-ème étage d'une $\mathbb{Z}_{p}$-extension de $\mathrm{K}$ contenue dans $\mathrm{K}_{S} / \mathrm{K}$. On veut déterminer toutes les $p$-extensions élémentaires $\mathrm{L}=$ $\mathrm{K}_{i}(\sqrt[p]{\alpha})$ de $\mathrm{K}_{i}$ qui correspondent au $i+1$-ème étage d'une $\mathbb{Z}_{p}$-extension de $\mathrm{K}_{S} / \mathrm{K}$.

Il y a deux volets. D'une part, il faut déterminer si $\mathrm{L}=\mathrm{K}_{i}(\sqrt[p]{\alpha})$ est dans le compositum $\widetilde{\mathrm{K}_{S}}$ des $\mathbb{Z}_{p}$-extensions de $\mathrm{K}$ contenues dans $\mathrm{K}_{S}$ : c'est le logarithme de Gras qui va permettre de répondre. D'autre part, il faut voir si L est l'étage d'une $\mathbb{Z}_{p}$-extension. Pour ce second point, on peut s'appuyer 
sur le lemme suivant, conséquence d'un résultat élémentaire à propos des modules sur les anneaux principaux.

Lemme 4.1. Soient $\mathrm{K} \subset \mathrm{L} \subset \widetilde{\mathrm{K}}_{S}$. Alors $\mathrm{L}$ est l'étage d'une $\mathbb{Z}_{p}$-extension de $\mathrm{K}$ si et seulement si, $\mathrm{L} / \mathrm{K}$ est cyclique.

Preuve. Un sens est évident. Réciproquement. Supposons $\mathrm{L} / \mathrm{K}$ cyclique. Soit $H$ le sous-groupe de $G=\operatorname{Gal}\left(\widetilde{\mathrm{K}}_{S} / \mathrm{K}\right)$ vérifiant $G / H \simeq \mathrm{Gal}(\mathrm{L} / \mathrm{K}) \simeq$ $\mathbb{Z} / p^{n} \mathbb{Z}$. Le $\mathbb{Z}_{p}$-module $H$ est de même rang $r$ que $G$. D'après la théorie des diviseurs élémentaires, il existe une $\mathbb{Z}_{p}$-base $\left\{e_{1}, \cdots, e_{r}\right\}$ de $G$ telle que $\left\{e_{1}, \cdots, e_{r-1}, p^{n} e_{r}\right\}$ soit aussi une base de $H$. Soit $H^{\prime}=\mathbb{Z}_{p} e_{1}+\cdots+\mathbb{Z}_{p} e_{r-1}$. Alors $H^{\prime} \subset H$. Le sous-corps de $\widetilde{\mathrm{K}}_{S}$ fixé par $H^{\prime}$ contient L et c'est aussi une $\mathbb{Z}_{p^{-}}$-extension de $\mathrm{K}$.

La recherche va alors se faire en trois étapes.

(i) Déterminer, à l'aide de la théorie de Kummer, toutes les extensions $p$-élémentaires de $\mathrm{K}_{i}$ contenues dans $\mathrm{K}_{S}$. Parmi celles-ci, trouver celles qui sont abéliennes puis cycliques sur $\mathrm{K}$;

(ii) Déterminer un conducteur $\mathfrak{f}$ de $\mathrm{K}$ tel que $\mathrm{K}_{\mathfrak{f}}$ contient toutes les $p$ extensions élémentaires de $\mathrm{K}_{i}$ abéliennes sur $\mathrm{K}$.

Trouver ensuite un système de Frobenius $\operatorname{frob}\left(\mathfrak{p}^{(1)}\right), \cdots, \operatorname{frob}\left(\mathfrak{p}^{(r)}\right)$ engendrant le groupe $\operatorname{Gal}\left(\mathrm{K}_{\mathfrak{f}} / \mathrm{K}_{i}\right) \subset \operatorname{Gal}\left(\mathrm{K}_{\mathfrak{f}} / \mathrm{K}\right.$ ) (il faut s'assurer que pour $i=1, \cdots, r, \mathfrak{p}^{(i)}$ est premier à $\left.p\right)$. Notons par $\Omega$ une telle famille.

Le lemme suivant est une application immédiate du théorème 4.1.

Lemme 4.2. Soit $\Omega(i+1)=$

$$
\left\{\mathfrak{b}=\prod_{i}^{r} \operatorname{frob}\left(\mathfrak{p}^{(i)}\right)^{a_{2}}, \mathfrak{p}^{(i)} \in \Omega, a_{i}=0, \cdots, p-1 \mid \log (\mathfrak{b}) \in p^{i+1} \log I_{\mathrm{K}, S}\right\}
$$

Alors le sous-corps maximal de $\mathrm{K}_{\mathfrak{f}}$ fixé par tous les éléments de $\Omega(i+1)$ est le compositum de tous les $i+1$-étages de $\widetilde{\mathrm{K}_{S}} / \mathrm{K}$.

(iii) Enfin, la proposition suivante permet de conclure.

Proposition 4.2. Soit $\mathrm{L}=\mathrm{K}_{i}(\sqrt[p]{\alpha}) / \mathrm{K}$ une sous-extension cyclique de $\mathrm{K}_{S} / \mathrm{K}$. Le corps $\mathrm{K}_{i}(\sqrt[p]{\alpha})$ est le $i+1$-étage d'une $\mathbb{Z}_{p}$-extension de $\widetilde{\mathrm{K}}_{S} / \mathrm{K}$ si et seulement si, pour tout $\mathfrak{b}=\prod_{i}^{r} \operatorname{frob}\left(\mathfrak{p}^{(i)}\right)^{a_{i}} \in \Omega(i+1)$,

$$
\prod_{j=1}^{r}\left(\frac{\alpha}{\mathfrak{P}^{(j)}}\right)^{a_{j}}=1,
$$

où $\mathfrak{P}^{(j)}, j=1, \cdots, r$ est un premier (quelconque) de $\mathrm{K}_{i}$ au-dessus de $\mathfrak{p}^{(j)}$. 
Preuve. Notons tout d'abord que par le choix de $\mathfrak{f}, \mathrm{L}$ est un sous-corps de $\mathrm{K}_{\mathfrak{f}}$. Pour $j=1, \cdots, r$, l'idéal $\mathfrak{p}^{(j)}$ est décomposé dans $\mathrm{K}_{i} / \mathrm{K}$. Ainsi, on a la propriété suivante du symbole d'Artin :

$$
\operatorname{frob}\left(\mathfrak{p}^{(j)}\right)=\left(\frac{\mathfrak{p}^{(j)}}{\mathrm{K}_{\mathfrak{f}} / \mathrm{K}}\right)=\left(\frac{\mathfrak{P}^{(j)}}{\mathrm{K}_{\mathfrak{f}} / \mathrm{K}_{i}}\right)
$$

pour n'importe quel idéal premier $\mathfrak{P}^{(j)}$ de $K_{i}$ au-dessus de $\mathfrak{p}^{(j)}$. Ensuite,

$$
\begin{aligned}
& \sigma_{\mathfrak{p}^{(j)} \mid \mathrm{L}}=\left(\frac{\mathfrak{P}^{(j)}}{\mathrm{L} / \mathrm{K}_{i}}\right) \text {. Ainsi, il vient : } \\
& \quad\left(\frac{\mathfrak{b}}{\mathrm{K}_{i}(\sqrt[p]{\alpha}) / \mathrm{K}_{i}}\right) \sqrt[p]{\alpha}=\prod_{j=1}^{r}\left(\frac{\mathfrak{P}^{(j)}}{\mathrm{K}_{i}(\sqrt[p]{\alpha}) / \mathrm{K}_{i}}\right)^{a_{j}} \sqrt[p]{\alpha}=\prod_{j=1}^{r}\left(\frac{\alpha}{\mathfrak{P}^{(j)}}\right)^{a_{j}} \sqrt[p]{\alpha} .
\end{aligned}
$$

Le lemme 4.2 permet finalement de conclure .

\section{5. À propos du théorème du miroir}

Choisissons maintenant $T$ et $S$ vérifiant $S \cup T=S_{p}$. Supposons toujours également que $\mathrm{K}_{S} / \mathrm{K}$ contient une $\mathbb{Z}_{p}$-extension $\mathrm{K}_{\infty} / \mathrm{K}$.

Nous désirons appliquer un résultat de dualité le long de $\mathrm{K}_{\infty} / \mathrm{K}$. Pour ce faire, nous allons supposer que $\mathrm{K}$ contient les racines $p$-èmes de l'unité. Il va alors être possible de donner des informations sur les invariants $\mathrm{r}_{S}$ et $\rho_{S}$ de $\mathcal{X}_{S}$ à partir du $\Lambda$-module $\mathcal{X}_{T}^{S}$. $\grave{A}$ noter que contrairement à la $\mathbb{Z}_{p^{-}}$ extension cyclotomique, $\mathrm{K}_{\infty} / \mathrm{K}$ ne va contenir qu'un nombre fini de racines de l'unités, nous empêchant d'obtenir théoriquement des renseignements sur l'invariant $\lambda_{S}$.

Ceci nous permettra de donner un critère de nullité de l'invariant $\mathrm{r}_{S}$. Celui-ci est adapté à la situation "déséquilibrée", c'est à dire lorsque $\delta_{S}$ est bien plus grand que $\delta_{T}$.

Pour l'étude d'une telle dualité dans le cas cyclotomique, nous renvoyons à $[15]$.

Proposition 5.1. Soit K un corps totalement imaginaire contenant les racines p-èmes de l'unité. Soit $\mathrm{K}_{\infty} / \mathrm{K}$ une $\mathbb{Z}_{p}$-extension contenue dans $\mathrm{K}_{S} / \mathrm{K}$. Notons par $\left(\rho_{S}^{T}, \mathrm{r}_{S}^{T}, \lambda_{T}^{S}\right)$ (respectivement $\left(\rho_{T}^{S}, \mathrm{r}_{T}^{S}, \lambda_{S}^{T}\right)$ ) les invariants $d u \Lambda$-module $\mathcal{X}_{S}^{T}$ (respectivement $\mathcal{X}_{T}^{S}$ ) relativement à $\mathrm{Gal}\left(\mathrm{K}_{\infty} / \mathrm{K}\right)$. Notons par $\tilde{s}$ (respectivement $\tilde{t}$ ) le nombre de places de $S$ (respectivement de $T$ ) totalement décomposées dans $\mathrm{K}_{\infty} / \mathrm{K}$. Il vient alors la formule suivante :

$$
\frac{\delta_{T}}{2}+\tilde{t}+\rho_{S}^{T}+\mathrm{r}_{S}^{T}=\frac{\delta_{S}}{2}+\tilde{s}+\rho_{T}^{S}+\mathrm{r}_{T}^{S} .
$$

Preuve. Nous allons utiliser une identité due à Gras que l'on peut trouver dans [7] (théorème 4.6, chapitre I). 
Proposition 5.2. Sous la condition $T \cup S=S_{p}$, nous avons pour chaque étage $\mathrm{K}_{n}$ la formule

$$
d_{p} G_{n, T}^{S}-d_{p} G_{n, S}^{T}=t_{n}-s_{n}+\delta_{T_{n}}-r_{2}\left(\mathrm{~K}_{n}\right)
$$

D'après les lemmes $2.1,2.4$ et 2.5 , asymptotiquement cette formule devient

$$
p^{n}\left(\rho_{T}^{S}+\mathrm{r}_{T}^{S}\right)-p^{n}\left(\rho_{S}^{T}+\mathrm{r}_{S}^{T}\right)=p^{n}(\tilde{t}-\tilde{s})+p^{n} \delta_{T}-p^{n} r_{2}+O(1)
$$

On conclut en notant que $2 r_{2}=\delta_{T}+\delta_{S}$.

Nous sommes en mesure de pouvoir donner un critère de nullité de l'invariant $\mathrm{r}_{S}$.

Corollaire 5.1. Sous les hypothèses de la proposition 5.1, il vient

$$
\mathrm{r}_{S}^{T} \leq \tilde{s}+\rho_{T}^{S}+\mathrm{r}_{T}^{S}
$$

Ainsi dès lors que $\tilde{t}=\tilde{s}=\rho_{T}^{S}=\mathrm{r}_{T}^{S}=0$, on a $\mathrm{r}_{S}=0$ et $\rho_{S}=1 / 2\left(\delta_{S}-\delta_{T}\right)=$ $\delta_{S}-r_{2}$.

Preuve. À l'étage $\mathrm{K}_{n}$, le $\mathbb{Z}_{p}$-rang du groupe $\operatorname{Gal}\left(\mathrm{K}_{n, S}^{T} / \mathrm{K}_{n}\right)$ est exactement égal à $\delta_{S_{n}}-r k_{\mathbb{Z}_{p}} \varphi_{S_{n}}\left(E_{\mathrm{K}_{n}}^{T}\right)$ et est donc minoré par $\delta_{S\left(\mathrm{~K}_{n}\right)}-\left(r_{2}\left(\mathrm{~K}_{n}\right)+t_{n}-1\right)$. Pour $n$ assez grand le $\mathbb{Z}_{p}$-rang de $\operatorname{Gal}\left(\mathrm{K}_{n, S}^{T} / \mathrm{K}_{n}\right)$ est donc minoré par :

$$
p^{n}\left(\delta_{S}\right)-p^{n}\left(r_{2}+\tilde{t}\right)+O(1)
$$

On a donc l'inégalité (avec le lemme 2.1)

$$
p^{n} \rho_{S}^{T} \geq p^{n} \delta_{S}-p^{n}\left(r_{2}+\tilde{t}\right)+O(1)
$$

qui permet d'obtenir

$$
\rho_{S}^{T} \geq \delta_{S}-r_{2}-\tilde{t}
$$

Reportant cette inégalité dans celle de la proposition 5.1 et en notant que $r_{2}=\delta^{S}+\delta^{T}$, on obtient bien :

$$
\mathrm{r}_{S}^{T} \leq \tilde{s}+\rho_{T}^{S}+\mathrm{r}_{T}^{S} .
$$

Lorsque $\tilde{s}=\rho_{T}^{S}=\mathrm{r}_{T}^{S}=0$, il vient $\mathrm{r}_{S}^{T}=0$. Maintenant si $\tilde{t}=0$, cela signifie qu'il n'y a qu'un nombre fini de places dans $\mathrm{K}_{\infty} / \mathrm{K}$ au-dessus des places de $T$. Or on passe de $\mathcal{X}_{S}$ à $\mathcal{X}_{S}^{T}$ en quotientant par les groupes de décomposition des places de $T$, chacun étant cyclique ou pro-cyclique. Ces groupes étant en nombre fini, le quotient ne change pas l'invariant $r$ et par conséquent $\mathrm{r}_{S}=\mathrm{r}_{S}^{T}=0$.

La formule de la proposition 5.1 montre alors que $\rho_{S}^{T}=1 / 2\left(\delta_{S}-\delta_{T}\right)$. La aussi, comme $\tilde{t}=0$, on conclut en notant que $\rho_{S}^{T}=\rho_{S}$. 
Remarque. Soit $\mathrm{K}_{n_{0}}$ un étage de $\mathrm{K}_{\infty} / \mathrm{K}$ où toutes les places inertes et ramifiées de $S_{p}$ dans $\mathrm{K}_{\infty} / \mathrm{K}$ le sont totalement dans $\mathrm{K}_{\infty} / \mathrm{K}_{n_{0}}$. Supposons qu'il existe $n \geq n_{0}$ tel que $d_{p} G_{n, T}^{S}=d_{p} G_{n+1, T}^{S}$. Alors, pour tout $m \geq n$, on a $d_{p} G_{n, T}^{S}=d_{p} G_{m, T}^{S}$. Utilisant ensuite le proposition 5.2, on obtient, pour tout $m \geq n, d_{p} G_{m, S}^{T}=-t_{m}+s_{m}-p^{m} \delta^{T}+p^{m} r_{2}+d_{p} G_{n, T}^{S}$.

Nous allons montrer que dans un certain sens, le corollaire 5.1 est optimal.

Mais avant d'énoncer le résultat, remarquons que la section 4, peut être développée dans un contexte un peu plus général, en considérant des pro- $p$ extensions $S$-ramifiées et $T$-décomposées (avec $T$ non nécessairement contenu dans $S_{p}$ ). D'après le lemme 2.4 , la lecture à chaque étage $\mathrm{K}_{n}$ du groupe $G_{n, S}^{T, a b}$ est alors relativement simple. Comme pour le cas $T=\emptyset$, il alors est naturel d'introduire les notions de $S-T$-conditions arithmétiques faibles et fortes, cohomologiques faibles et fortes. Les résultats de la section 4 restent alors parfaitement valables dans ce cadre.

Revenons au contexte de cette section. On suppose donc $S \cup T=S_{p}$, mais également que pour une $\mathbb{Z}_{p}$-extension $\mathrm{K}_{\infty} / \mathrm{K}$ contenue dans $\mathrm{K}_{S}$, il vient $T=\widetilde{T}$.

Cela signifie en particulier que la quantité $\delta_{S}-\mathrm{rk}_{\mathbb{Z}_{p}} \varphi\left(E_{\mathrm{K}}^{T}\right)$ n'est pas nulle.

Alors sous ces conditions, on a la proposition suivante :

Proposition 5.3. Supposons le morphisme $\varphi_{S}: \mathbb{Z}_{p} \otimes E_{\mathrm{K}}^{T} \rightarrow U_{S}^{1}$ injectif (c'est ce que l'on peut appeler la $S-T$ condition arithmétique forte). Alors,

$$
\mathrm{r}_{S}=\mathrm{r}_{S}^{T}=\mathrm{r}_{T}^{S}+\rho_{T}^{S}+\widetilde{s}
$$

Preuve. La $S-T$-condition arithmétique est satisfaite pour le corps $\mathrm{K}$. Cela signifie que pour toute $\mathbb{Z}_{p}$-extension $\mathrm{K}_{\infty} / \mathrm{K}$ contenue dans $\mathrm{K}_{S}^{T} / \mathrm{K}$, la $S-T$ condition arithmétique faible est vérifiée, à savoir : $\rho_{S}^{T}=\delta_{S}-\left(r_{1}+r_{2}+t\right)$, ceci d'après les propositions 3.1, 3.2 et le lemme 3.2. Il en est de même pour la $S$-condition arithmétique faible le long de toute $\mathbb{Z}_{p}$-extension contenue dans $\mathrm{K}_{S} / \mathrm{K}: \rho_{S}=\delta_{S}-\left(r_{1}+r_{2}\right)$.

Ainsi, pour une $\mathbb{Z}_{p}$-extension $\mathrm{K}_{\infty} / \mathrm{K}$ contenue dans $\mathrm{K}_{S}^{T} / \mathrm{K}$, la proposition 5.1 nous donne exactement $: \mathrm{r}_{S}^{T}=\rho_{T}^{S}+\mathrm{r}_{T}^{S}+\widetilde{s}$.

De plus,

$$
\begin{aligned}
& \operatorname{rk}_{\mathbb{Z}_{p}} \operatorname{Gal}\left(\mathrm{K}_{n, S}^{T} / \mathrm{K}_{n}\right)^{a b}=p^{n}\left(\rho_{S}-t\right)+O(1) \\
& \operatorname{rk}_{\mathbb{Z}_{p}} \operatorname{Gal}\left(\mathrm{K}_{n, S} / \mathrm{K}_{n}\right)^{a b}=p^{n} \rho_{S}+O(1) .
\end{aligned}
$$

Il vient ainsi :

$$
\operatorname{rk}_{\mathbb{Z}_{p}}<D_{n, \mathfrak{P}}, \mathfrak{P} \in T_{n}>=p^{n} t+O(1)
$$


où $D_{n, \mathfrak{P}}$ est le groupe de décomposition de $\mathfrak{P}$ dans l'extension $\mathrm{K}_{n, S}^{a b} / \mathrm{K}_{n}$. Les groupes $D_{n, \mathfrak{P}}$ étant procyclique, il vient $d_{p}<D_{n, \mathfrak{P}}, \mathfrak{P} \in T_{n}>=p^{n} t+O(1)$.

Pour finir, l'égalité $\left(\rho_{S}^{T}+\mathrm{r}_{S}^{T}\right) p^{n}=\left(\rho_{S}+\mathrm{r}_{S}\right) p^{n}-d_{p}\left\langle D_{n, \mathfrak{P}}, \mathfrak{P} \in T_{n}\right\rangle$ $+O(1)$, devient après simplification :

$$
\mathrm{r}_{S}^{T}=\mathrm{r}_{S}-t+1 / p^{n} d_{p}<D_{n, \mathfrak{P}}, \mathfrak{P} \in T_{n}>+1 / p^{n} O(1)
$$

d'où $\mathrm{r}_{S}^{T}=\mathrm{r}_{S}$.

Cette proposition indique donc que pour montrer que l'invariant $\mathrm{r}_{S}$ est trivial, il est nécessaire d'effectuer un choix judicieux de la $\mathbb{Z}_{p}$-extension $\mathrm{K}_{\infty} / \mathrm{K}$. L'exemple 6.2 illustrera ceci.

\section{Deux exemples}

Dans cette section, nous allons détailler deux exemples. Tout d'abord, présentons notre démarche.

On se donne un corps de nombres $\mathrm{K}$, un nombre premier $p$ et un ensemble fini $S$ de places de K contenu dans $S_{p}$.

- Dans un premier temps, le logarithme de Gras nous permet de privilégier une $\mathbb{Z}_{p}$-extension $\mathrm{K}_{\infty}$ de $\mathrm{K}$.

- Ensuite, on vérifie que K satisfait la $S$-condition arithmétique forte. $\mathrm{Si}$ c'est le cas, le corollaire 3.2 et la proposition 3.2 montrent que l'invariant $\rho_{S} \mathrm{du} \mathbb{Z}_{p}\left[\left[\mathrm{Gal}\left(\mathrm{K}_{\infty} / \mathrm{K}\right)\right]\right]$-module $\mathcal{X}_{S}$ est exactement $\delta_{S}-\left(r_{1}+r_{2}\right)$.

- Dans un contexte favorable, le corollaire de dualité 5.1 permet de montrer que $\mu_{S}=0$. La proposition 3.4 indique alors que la dimension cohomologique de $G_{S}$ est au plus 2 .

- Maintenant, si le corollaire 5.1 ne s'applique pas, on peut essayer la proposition plus fine 3.9. Dans ce cas, il faut s'assurer que la $S$-condition arithmétique est satisfaite le long de $\mathrm{K}_{\infty} / \mathrm{K}$. A noter que ce corollaire peut également servir à montrer que l'invariant $\lambda_{S}$ est trivial.

- Le résultat de dualité de la remarque 5 peut permettre de donner une majoration de la quantité $\mathrm{rk}_{\mathbb{Z}_{p}} G_{n, S}-p^{n} \rho_{S}$ le long de $\mathrm{K}_{\infty} / \mathrm{K}$. Si la situation est bien choisie, cette majoration ne dépendra pas de $n$.

- Grâce au corollaire 3.3, on s'assure, après un nombre fini de calculs, que la $S$-condition arithmétique (ou cohomologique) est vérifiée le long de $\mathrm{K}_{\infty} / \mathrm{K}$. Si tel est le cas, une partie des hypothèses de la proposition 3.9 est réunie.

- Enfin, si la partie de torsion de $G_{n, S}^{a b}$ ne grandit pas trop vite, la proposition 3.9 s'applique et $\mu_{S}$ est trivial.

6.1. Prenons $\mathrm{K}=\mathbb{Q}(\theta)$, où $\theta$ vérifie $\theta^{4}+\theta^{3}+8 \theta^{2}-4 \theta+2=0$.

La signature de $\mathrm{K}$ est $(0,2)$. L'anneau des entiers $\mathcal{O}_{\mathrm{K}}$ de $\mathrm{K}$ est monogène : $\mathcal{O}_{K}=\mathbb{Z}[\theta] ; E_{\mathrm{K}}=< \pm 1, \varepsilon:=207 \theta^{3}-383 \theta^{2}+190 \theta-65>$. Le discriminant de $\mathcal{O}_{\mathrm{K}}$ vaut $132692=2^{2} \cdot 7^{2} \cdot 677$. 
Enfin, le groupe des classes de $\mathrm{K}$ est d'ordre 2, il est engendré par un idéal $\mathfrak{p}_{5}$ au-dessus de 5 .

Il y a exactement deux places de $\mathrm{K}$ au-dessus de $2: \mathfrak{p}_{2}^{(1)}=(\theta)$ et $\mathfrak{p}_{2}^{(2)}=$ $(2,1+\theta)=\left(17+3 \theta+2 \theta^{2}\right)$. L'indice de ramification de $\mathfrak{p}_{2}^{(1)}$ est 3.

Prenons $p=2$.

Exemple. Soit $\mathrm{K}=\mathbb{Q}(\theta)$ avec $\theta$ vérifiant $\theta^{4}+\theta^{3}+8 \theta^{2}-4 \theta+2=0$. Posons $S=\{(\theta)\}$. Il existe une $\mathbb{Z}_{2}$-extension $\mathrm{K}_{\infty}$ de $\mathrm{K}$ contenue dans $\mathrm{K}_{S} / \mathrm{K}$ telle que

(i) $\mathrm{K}_{S} / \mathrm{K}_{\infty}$ est libre ;

(ii) le $\Lambda$-module $H_{S}^{a b}=\operatorname{Gal}\left(\mathrm{K}_{S} / \mathrm{K}_{\infty}\right)^{a b}$ est un sous- $\Lambda$-module de $\Lambda:=$ $\mathbb{Z}_{p}\left[\left[\mathrm{Gal}\left(\mathrm{K}_{\infty} / \mathrm{K}\right)\right]\right]$ d'indice fini $\left(\rho_{S}=1\right.$ et $\left.\mu_{S}=\lambda_{S}=0\right)$.

De plus, la dimension cohomologique de $G_{S}$ est exactement 2 .

L'ensemble des calculs vont être effectués dans $\mathrm{K}_{\mathfrak{p}_{2}^{(1)}}: \pi=\theta$ est une uniformisante de $\mathrm{K}_{\mathfrak{p}_{2}^{(1)}}$; on notera par $\mathrm{v}=\mathrm{v}_{\pi}$ la valuation.

On sait tout d'abord que $U_{\mathfrak{p}_{2}^{(1)}}^{1} \simeq \pm 1 \oplus(1+\pi)^{\mathbb{Z}_{2}} \oplus\left(1+\pi^{5}\right)^{\mathbb{Z}_{2}} \oplus\left(1+\pi^{6}\right)^{\mathbb{Z}_{2}}$, voir par exemple [13].

On obtient ainsi la décomposition suivante de l'unité fondamentale :

$$
\varepsilon=(1+\pi)^{2+\cdots}\left(1+\pi^{5}\right)^{1+\cdots}\left(1+\pi^{6}\right)^{2+\cdots}
$$

Rappelons ensuite que le logarithme défini par Gras donne l'isomorphisme suivant

$$
\operatorname{Gal}\left(\widetilde{\mathrm{K}_{S}} / \mathrm{K}\right) \simeq \log U_{\mathfrak{p}_{2}^{(1)}}^{1}+\mathbb{Q}_{2} \log (\varepsilon) \bmod \mathbb{Q}_{2} \log (\varepsilon),
$$

où $\log$ désigne le logarithme 2 -adique dans $\mathrm{K}_{\mathfrak{p}_{2}^{(1)}}$. (on identifiera log et Log).

Le groupe des classes de $\mathrm{K}$ est engendré par un premier $\mathfrak{p}_{5}$ au-dessus de $5: \mathfrak{p}_{5}=(5,3+\theta)$. Il vient $\mathfrak{p}_{5}^{2}=\left(x_{5}\right)$ avec $x_{5}=3-8 \theta-\theta^{2}-\theta^{3}$ et finalement

$$
x_{5}=(1+\pi)^{2+\cdots}\left(1+\pi^{5}\right)^{4(\cdots)}\left(1+\pi^{6}\right)^{4(\cdots)}
$$

Ainsi clairement $\log \left(\mathfrak{p}_{5}\right)=1 / 2 \log \left(x_{5}\right) \in \log U_{\mathfrak{p}_{2}^{(1)}}^{1}$.

Par conséquent,

$$
\left(1 / 2 \log \left(x_{5}\right)+\log U_{\mathfrak{p}_{2}^{(1)}}^{1}+\mathbb{Q}_{2} \log (\varepsilon): \log U_{\mathfrak{p}_{2}^{(1)}}^{1}+\mathbb{Q}_{2} \log (\varepsilon)\right)=1
$$

Il y a donc disjonction linéaire entre $\mathrm{K}^{H}$ et $\widetilde{\mathrm{K}}_{S}$ (cf. théorème 4.1 ).

Notons ensuite que $x_{5}=\left(1+\theta+\theta^{2}\right)^{2}-4\left(2-2 \theta+9 \theta^{2}+5 \theta^{3}\right)$. Ainsi l'extension quadratique $\mathrm{K}\left(\sqrt{x_{5}}\right) / \mathrm{K}$ est non-ramifiée. 
Un calcul élémentaire montre que l'ordre de la torsion de $G_{S}^{a b}$ est 2 (celuici vaut $\left.2 \cdot \# \operatorname{Tor}_{\mathbb{Z}_{p}}\left(\log U_{S}^{1} / \log \left(E_{\mathrm{K}}\right)\right)\right)$.

Notons enfin que le morphisme $\varphi_{S}$ est (trivialement) injectif et ainsi l'extension $\mathrm{K}_{S} / \mathrm{K}$ contient deux $\mathbb{Z}_{2}$-extensions linéairement indépendantes. On peut résumer tout ceci dans le

Lemme 6.1. (i) $G_{S}^{a b} \simeq \mathbb{Z}_{2} \times \mathbb{Z} / 2 \mathbb{Z}$.

(ii) $\mathrm{K}\left(\sqrt{x_{5}}\right)$ est le corps de Hilbert $\mathrm{K}^{H}$ de $\mathrm{K}$. Il est linéairement disjoint de $\widetilde{\mathrm{K}_{S}}$.

6.1.1. Les $\mathbb{Z}_{2}$-extensions de $\mathrm{K}_{S} / \mathrm{K}$. Comme $\mathfrak{p}_{2}^{(2)}=\left(17+3 \theta+2 \theta^{2}\right)$, le groupe de décomposition $D_{\mathfrak{p}_{2}^{(2)}}$ de $\mathfrak{p}_{2}^{(2)}$ dans $\operatorname{Gal}\left(\widetilde{\mathrm{K}}_{S} / \mathrm{K}\right)$ correspond, via le logarithme de Gras, à $\mathbb{Z}_{2} \log \left(17+3 \theta+2 \theta^{2}\right)$ dans $\mathbb{Q}_{2} \log U_{\mathfrak{p}_{2}^{(1)}}^{1}+\mathbb{Q}_{2} \log \varepsilon$ $\bmod \mathbb{Q}_{2} \log \varepsilon$.

De l'égalité $17+3 \theta+2 \theta^{2}=(1+\pi)^{1+\cdots}\left(1+\pi^{5}\right)^{2+\cdots}\left(1+\pi^{6}\right)^{1+\cdots}$, on en déduit que le groupe $D_{\mathfrak{p}_{2}^{(2)}}$ est engendré par $a_{1} \log (1+\pi)+2 a_{2} \log \left(1+\pi^{5}\right)+$ $a_{3} \log \left(1+\pi^{6}\right)$ dans $\mathbb{Z}_{2} \log U_{\mathfrak{p}_{2}^{(1)}}^{1}+\mathbb{Q}_{2} \log \varepsilon \bmod \mathbb{Q}_{2} \log \varepsilon$, avec $a_{i} \in \mathbb{Z}_{2}^{\times}$. Posons $x_{2}=17+3 \theta+2 \theta^{2}$.

Remarquons ensuite que le premier 43 se décompose dans $\mathrm{K}$ en produit de trois idéaux premiers principaux (non-ramifiés) dont l'un, noté $\mathfrak{p}_{43}$, est de degré résiduel $1: \mathfrak{p}_{43}=\left(-1+17 \theta+3 \theta^{2}+2 \theta^{3}\right)$. Posons $x_{43}=-1+$ $17 \theta+3 \theta^{2}+2 \theta^{3}$.

On note ensuite que $x_{43}=(1+\pi)^{1+4+\cdots}\left(1+\pi^{5}\right)^{1+\cdots}\left(1+\pi^{6}\right)^{2+\cdots}$. Ainsi

$$
\log \left(x_{43}\right)=b_{1} \log (1+\pi)+b_{2} \log \left(1+\pi^{5}\right)+2 b_{3} \log \left(1+\pi^{6}\right)
$$

avec $b_{i} \in \mathbb{Z}_{2}^{\times}$.

La famille $\left\{\log x_{2}, \log x_{43}, \log (1+\pi)\right\}$ constitue une base de $\log U_{\mathfrak{p}_{2}(1)}^{1}$ (il suffit de noter que le déterminant de la matrice de passage est dans $\left.\mathbb{Z}_{2}^{\times}\right)$. Ensuite, il est facile de voir que le $\mathbb{Z}_{2}$-rang de $<\log x_{43}, \log x_{2}>$ $+\mathbb{Q}_{2} \log \varepsilon \bmod \mathbb{Q}_{2} \log \varepsilon$ vaut 2 .

Montrons pour finir que $\log (1+\pi) \in<\log x_{43}, \log x_{2}>+\mathbb{Q}_{2} \log \varepsilon$. Supposons qu'il existe une puissance de 2 non triviale et minimale, $2^{m}$, telle que $2^{m} \log (1+\pi)=\alpha \log x_{43}+\beta \log x_{2}+\gamma \log \varepsilon$, avec $\alpha, \beta, \gamma \in \mathbb{Z}_{2}$. En regardant la composante en $\log \left(1+\pi^{6}\right)$, on trouve $\beta \equiv 0 \bmod 2$. Reportant dans l'équation issue de la composante en $\log (1+\pi)$, on en déduit $\alpha \equiv 0 \bmod 2$, puis finalement $\gamma \equiv 0 \bmod 2$, ce qui contredit la minimalité de $m$. Ainsi $\log (1+\pi) \in<\log x_{43}, \log x_{2}>+\mathbb{Q}_{2} \log \varepsilon$. 
On vient donc de montrer que $\operatorname{Gal}\left(\widetilde{\mathrm{K}_{S}} / \mathrm{K}\right)=D_{\mathfrak{p}_{2}^{(2)}} \times<\operatorname{frob}\left(\mathfrak{p}_{43}\right)>$. Posons

$$
\mathrm{K}_{\infty}=\widetilde{\mathrm{K}}_{S}<\operatorname{frob}\left(\mathfrak{p}_{43}\right)>
$$

Le sous-corps $\mathrm{K}_{\infty}$ de $\mathrm{K}_{S}$ est une $\mathbb{Z}_{2}$-extension de $\mathrm{K}$ dans laquelle $\mathfrak{p}_{2}^{(2)}$ est totalement inerte. La place $\mathfrak{p}_{2}^{(1)}$ est totalement ramifiée dans $\mathrm{K}_{\infty} / \mathrm{K}$. Ainsi, pour $\mathrm{K}_{\infty} / \mathrm{K}, \tilde{t}=\tilde{s}=0$.

6.1.2. Le premier étage de $\mathrm{K}_{\infty} / \mathrm{K}$.

Lemme 6.2. Les extensions quadratiques de $\mathrm{K}$ contenues dans $\mathrm{K}_{S} / \mathrm{K}$ sont les extensions $\mathrm{K}(\sqrt{\theta}), \mathrm{K}(\sqrt{\varepsilon}), \mathrm{K}\left(\sqrt{x_{5}}\right)$ ainsi que leurs compositums. Les extensions quadratiques de $\mathrm{K}$ contenues dans $\widetilde{\mathrm{K}}_{S} / \mathrm{K}$ sont : $\mathrm{K}\left(\sqrt{\varepsilon \cdot x_{5}}\right)$, $\mathrm{K}(\sqrt{\theta}), \mathrm{K}\left(\sqrt{\theta \cdot \varepsilon \cdot x_{5}}\right)$. Le premier étage de la $\mathbb{Z}_{2}$-extension $\mathrm{K}_{\infty} / \mathrm{K}$ est le corps $\mathrm{K}\left(\sqrt{\theta \cdot \varepsilon \cdot x_{5}}\right)$.

Preuve. On peut noter que $x, \varepsilon \equiv 1 \bmod \left(\mathfrak{p}_{2}^{(2)}\right)^{2}$. Les extensions $\mathrm{K}(\sqrt{\theta})$, $\mathrm{K}(\sqrt{\varepsilon})$ sont donc $S$-ramifiées.

On veut ensuite déterminer le $p$-radical de $\widetilde{\mathrm{K}_{S}} / \mathrm{K}$. Tout va se passer dans le corps de rayon $K_{\mathfrak{m}}$ de $K$ pour $\mathfrak{m}=\left(\theta^{7}\right)$.

Un calcul montre : $\mathrm{Cl}_{\mathrm{K}, \mathfrak{m}}=\mathbb{Z} / 4 \times(\mathbb{Z} / 2)^{2}=<\mathrm{Cl}\left(\mathfrak{p}_{5}\right), \mathrm{Cl}\left(\mathfrak{p}_{5}^{\prime}\right), \mathrm{Cl}\left(\mathfrak{p}_{7}\right)>$, où $\mathfrak{p}_{5}, \mathfrak{p}_{5}^{\prime}, \mathfrak{p}_{7}$ sont des idéaux premiers au-dessus de 5 et 7 .

Il faut alors déterminer le développement de ces générateurs dans $U_{\mathfrak{p}_{2}^{(1)}}$ :

- $\mathfrak{p}_{5}^{2}=\left(x_{5}:=3-8 \theta-\theta^{2}-\theta^{3}\right) ; x_{5}=(1+\pi)^{2+\cdots}\left(1+\pi^{5}\right)^{4(\cdots)}\left(1+\pi^{6}\right)^{4(\cdots)}$;

- $\mathfrak{p}_{5}^{\prime 2}=\left(x_{5}^{\prime}:=-5+8 \theta+\theta^{2}+\theta^{3}\right) ; \varepsilon x_{5}^{\prime}=(1+\pi)^{16+\cdots}\left(1+\pi^{5}\right)^{8+\cdots}$ $\left(1+\pi^{6}\right)^{2+\cdots}$

- $\mathfrak{p}_{7}^{2}=\left(x_{7}:=-1+2 \theta-3 \theta^{2}-\theta^{3}\right) ; \varepsilon x_{7}=(1+\pi)^{4+\cdots}\left(1+\pi^{5}\right)^{2+\cdots}$ $\left(1+\pi^{6}\right)^{4+\cdots}$

De l'algèbre linéaire élémentaire dans $\mathbb{F}_{2}$ montre que seul $\log \left(\mathfrak{p}_{7}\right) \in$ $2 \log I_{K, S}$ (ici, $\Omega(1)=\left\{\mathfrak{p}_{7}\right\}$ ). Ainsi le radical des 2-extensions élémentaires de $\widetilde{\mathrm{K}}_{S} / \mathrm{K}$ est fixé par le frobenius de $\mathfrak{p}_{7}$.

Comme $\left(\frac{\varepsilon}{\mathfrak{p}_{7}}\right)=\left(\frac{x_{5}}{\mathfrak{p}_{7}}\right)=\left(\frac{3}{7}\right)-1,\left(\frac{\theta}{\mathfrak{p}_{7}}\right)=\left(\frac{4}{7}\right)=1$, concernant le radical, on conclut facilement.

Pour finir, rappelons que la place $\mathfrak{p}_{43}=\left(-1+17 \theta+3 \theta^{2}+2 \theta^{3}\right)$ doit être décomposée dans $\mathrm{K}_{1} / \mathrm{K}$. Comme $\theta \equiv 35 \bmod \mathfrak{p}_{43}, \varepsilon \equiv 32 \bmod \mathfrak{p}_{43}$ et $x_{5} \equiv 26 \bmod \mathfrak{p}_{43}$, il vient, $\left(\frac{\theta}{\mathfrak{p}_{43}}\right)=-1,\left(\frac{\varepsilon}{\mathfrak{p}_{43}}\right)=+1$, et $\left(\frac{x_{5}}{\mathfrak{p}_{43}}\right)=-1$, d'où $\mathrm{K}_{1}=\mathrm{K}\left(\sqrt{\theta \cdot \varepsilon \cdot x_{5}}\right)$. 
6.1.3. Les $\Lambda$-modules $\mathcal{X}_{T}^{S}$ et $\mathcal{X}_{S}^{T}$. On peut calculer directement la torsion de $G_{1, S}^{a b}$ et noter que celle-ci est d'ordre 2 . Si l'on montre que la $S$-condition cohomologique est satisfaite le long de $\mathrm{K}_{\infty} / \mathrm{K}$, alors la proposition 3.9 peut s'appliquer, pour obtenir $\mathrm{r}_{S}=\lambda_{S}=0$, c'est à dire $H_{S}^{a b} \hookrightarrow \Lambda$.

D'autre part, d'après le corollaire 3.4 , on a $\operatorname{cd}\left(G_{S}\right) \leq 2$. Comme le groupe $G_{S}^{a b}$ a de la torsion, $G_{S}$ n'est pas libre est ainsi $\operatorname{cd}\left(G_{S}\right)=2$.

Il reste donc à s'assurer que l'on peut appliquer la proposition 3.9.

La stratégie va être la suivante. Nous allons d'abord contrôler le rang du défaut de la $S$-condition arithmétique le long de $\mathrm{K}_{\infty} / \mathrm{K}$. Pour ce faire, nous allons utiliser la dualité le long de $\mathrm{K}_{\infty} / \mathrm{K}$. Puis, nous appliquerons le corollaire 3.3.

Lemme 6.3. On a l'égalité suivante :

$$
d_{2} G_{T}^{S}=d_{2} G_{1, T}^{S}=1
$$

Preuve. D'après la proposition 5.2, nous avons

$$
d_{p} G_{T}^{S}=d_{p} G_{S}^{T}-1
$$

D'autre part, $G_{S}^{a b} \simeq \mathbb{Z}_{2}^{2} \times \mathrm{Cl}_{\mathrm{K}}$. Comme $\mathfrak{p}_{2}^{(2)}$ est inerte dans $\mathrm{K}_{\infty} / \mathrm{K}$, on a $d_{2} G_{S}^{T}=2$, d'où $d_{2} G_{T}^{S}=1$.

$\mathrm{Au}$ niveau du second étage, la proposition 4.1 indique

$$
d_{p} G_{1, T}^{S}=d_{p} G_{1, S}^{T}-2
$$

Après calcul, on a : $d_{2} G_{1, S}=4$. Comme la place de $T$ est inerte dans $\mathrm{K}_{\infty} / \mathrm{K}$, il vient $: d_{2} G_{1, S}^{T}=4-1=3$. Par conséquent, $d_{2} G_{1, T}^{S}=1$.

A noter que l'on obtient dès ici $\mathrm{r}_{T}^{S}=\rho_{T}^{S}=0$, ceci par la proposition 2.8. En fait, le module $\mathcal{X}_{T}^{S}$ est de 2-rang égal à 1 et ainsi pour tout $n$, $d_{2} G_{n, T}^{S}=1$.

Utilisant la formule de la remarque 5 , on en déduit $d_{2} G_{n, S}^{T}=2^{n}+1$, et ce pour tout entier $n$. L'extension $\mathrm{K}_{\infty} / \mathrm{K}$ étant $S$-ramifiée mais pas $T$-décomposée, on obtient que pour tout $n, d_{2} G_{n, S}=2^{n}+2\left(\operatorname{car} t_{n}=1\right)$.

On va appliquer le corollaire 3.3. Pour cela, on note que $\mathrm{rk}_{\mathbb{Z}_{2}} G_{n, S}^{a b}-\rho_{S}{ }^{n} \leq$ 2. Ainsi le polynôme caractéristique $P_{S}$ de $\mathcal{X}_{S}$ ne peut-être divisible que par $X$ ou bien par $X+2$. Il faut donc vérifier que la $S$-condition arithmétique est satisfaite pour $\mathrm{K}$ et $\mathrm{K}_{1}$. Pour $\mathrm{K}$, c'est déja fait. Pour $\mathrm{K}_{1}=\mathrm{K}(\alpha)$, avec $\alpha^{8}+7 \alpha^{6}+26 \alpha^{4}+56 \alpha^{2}+\alpha=0$, on note que $E_{\mathrm{K}_{1}}=< \pm 1>\times$ $<\varepsilon_{1}, \varepsilon_{2}, \varepsilon_{2}>$, tel que : $\varepsilon_{1} \equiv 1+\pi^{11}+\pi^{13} \bmod \left(\pi^{15}\right), \varepsilon_{2} \equiv 1+\pi^{11} \bmod \left(\pi^{15}\right)$ $\varepsilon_{5} \equiv 1+\pi^{2}+\pi^{5}+\pi^{10}+\pi^{12} \bmod \left(\pi^{1} 5\right)$, où $\pi$ est une uniformisante du complété de $K_{1}$ en l'unique place $\mathfrak{P}$ de $K_{1}$ au-dessus de $\mathfrak{p}_{2}^{(1)}$. 
Si $\prod_{i} \varepsilon_{i}^{a_{i}}=1$ est une relation linéaire entre les unités fondamentales $\varepsilon_{i}$ de $\mathrm{K}_{1}$, avec $a_{i} \in \mathbb{Z}_{2}$, il suffit de la regarder modulo $\pi^{13}$, pour obtenir $a_{i}=0$, quelque soit $i=1,2,3$. La condition arithmétique est donc vérifiée pour $\mathrm{K}_{1}$. Le corollaire 3.3 s'applique. La $S$-condition cohomologique est vérifiée le long de $\mathrm{K}_{\infty} / \mathrm{K}$.

Pour finir, notons que le corps $\mathrm{K}_{1} \mathrm{~K}^{H}$ est absorbé dans une $\mathbb{Z}_{2}$-extension de $\mathrm{K}_{1}$.

6.2. L'exemple qui vient illustre la proposition 5.3.

Soit le corps $\mathrm{K}=\mathbb{Q}(\theta)$, où $\theta$ vérifie $\theta^{10}+2 \theta^{9}+2 \theta^{8}+\theta^{7}+2=0$.

Le corps $\mathrm{K}$ est totalement imaginaire. L'anneau des entiers est $\mathcal{O}_{\mathrm{K}}=$ $\mathbb{Z}[\theta], E_{\mathrm{K}}= \pm 1<\varepsilon_{1}, \varepsilon_{2}, \varepsilon_{3}, \varepsilon_{4}>$, avec $\varepsilon_{1}=\theta^{7}+\theta^{6}+\theta^{5}+\theta^{4}-1, \varepsilon_{2}=\theta^{7}+$ $2 \theta^{6}+2 \theta^{5}+\theta^{4}+\theta^{3}+\theta^{2}-1, \varepsilon_{3}=\theta^{9}+\theta^{8}+1$ et $\varepsilon_{4}=\theta^{9}+\theta^{7}-\theta^{6}+\theta^{5}-\theta^{4}+\theta^{3}+1$.

L'ideal $2 \mathcal{O}_{\mathrm{K}}$ se factorise comme suit : $2 \mathcal{O}_{\mathrm{K}}=\mathfrak{p}_{2}^{(1)}{ }^{7} \mathfrak{p}_{2}^{(2)} \mathfrak{p}_{4}$, où $\mathfrak{p}_{4}$ est un idéal premier de degré résiduel 2.

Posons $S=\left\{\mathfrak{p}_{2}^{(1)}, \mathfrak{p}_{2}^{(2)}\right\}$ et $T=\left\{\mathfrak{p}_{4}\right\}$.

On peut alors vérifier que $G_{S}^{a b} \simeq \mathbb{Z}_{2}^{4} \times \mathbb{Z} / 2 \mathbb{Z}$. En particulier, $\mathrm{K}$ vérifie la $S$-condition arithmétique.

Exemple. Soit $\mathrm{K}(\theta)$ avec $\theta$ vérifiant $\theta^{10}+2 \theta^{9}+2 \theta^{8}+\theta^{7}+2=0$. Posons $S=\{(\theta),(1+\theta)\}$.

(i) Il existe une $\mathbb{Z}_{2}$-extension $\mathrm{K}_{\infty} / \mathrm{K}$ contenue dans $\mathrm{K}_{S} / \mathrm{K}$ où le $\mathbb{Z}_{2}\left[\left[\mathrm{Gal}\left(\mathrm{K}_{\infty} / \mathrm{K}\right)\right]\right]-$ module $H_{S}^{a b}:=\operatorname{Gal}\left(\mathrm{K}_{S} / \mathrm{K}_{\infty}\right)^{a b}$ a pour invariant $\rho_{S}=3$ et $\mu_{S}=\lambda_{S}=0$.

(ii) Le groupe $\mathrm{Gal}\left(\mathrm{K}_{S} / \mathrm{K}_{\infty}\right)$ est libre et la dimension cohomologique de $G_{S}$ est exactement 2 .

(iii) Il existe une $\mathbb{Z}_{2}$-extension $\mathrm{K}_{\infty}^{\prime} / \mathrm{K}$ contenue dans $\mathrm{K}_{S} / \mathrm{K}$ où le $\mathbb{Z}_{2}\left[\left[\mathrm{Gal}\left(\mathrm{K}_{\infty}^{\prime} / \mathrm{K}\right)\right]\right]$-module $H_{S}^{\prime a b}$ a pour invariant $\rho_{S}^{\prime}=3$ et $\mu_{S}^{\prime}=1$. Le groupe $\mathrm{Gal}\left(\mathrm{K}_{S} / \mathrm{K}_{\infty}^{\prime}\right)$ est de dimension cohomologique 2 .

Détaillons succintement cet exemple.

6.2.1. Une première $\mathbb{Z}_{2}$-extension. Le logarithme de Gras permet ensuite de montrer que le corps $K_{1}=K\left(\sqrt{\theta^{2}+\theta}\right)$, de degré 20 sur $\mathbb{Q}$, est le premier étage d'une $\mathbb{Z}_{2}$-extension de $\mathrm{K}$ contenue dans $\mathrm{K}_{S} / \mathrm{K}$. Soit $\mathrm{K}_{\infty}$ une telle $\mathbb{Z}_{2}$ extension. Pour cette $\mathbb{Z}_{2}$-extension, l'invariant $\rho_{S}$ de $\mathcal{X}_{S}:=\operatorname{Gal}\left(\mathrm{K}_{S} / \mathrm{K}_{\infty}\right)^{a b}$ est donc égal à $\delta_{S}-\left(r_{1}+r_{2}\right)$ c'est à dire à 3 .

On vérifie dans un premier temps que $d_{2} G_{T}=d_{2} G_{1, T}=0$. 
D'après la proposition 2.8, $\mathcal{X}_{T}=0$. Il en est donc de même pour $\mathcal{X}_{T}^{S}$.

D'après la remarque 5 , pour tout $n, d_{2} G_{n, S}^{T}=-t_{n}+s_{n}-2 \cdot 2^{n}+5 \cdot 2^{n}$. Ainsi, $d_{2} G_{n, S}-2^{n} \rho_{S} \leq d_{2} G_{n, S}^{T}+t_{n} \leq 2$.

D'après la proposition 3.1 , la condition arithmétique est satisfaite le long de $K_{\infty} / K$ si et seulement si, elle l'est pour les corps $K$ et $K_{1}$, ce que l'on peut vérifier.

Pour $\mathrm{K}_{1}$, on note ensuite que le 2 -rang de $\operatorname{Tor}_{\mathbb{Z}_{2}}\left(G_{1, S}^{a b}\right)$ est égal à 1.

La proposition 3.9 s'applique et ainsi $\mathrm{r}_{S}=0$.

Par conséquent, le groupe $\operatorname{Gal}\left(\mathrm{K}_{S} / \mathrm{K}_{\infty}\right)$ est libre et donc $\operatorname{cd}\left(G_{S}\right)=2$.

6.2.2. Une seconde $\mathbb{Z}_{2}$-extension. Posons ensuite $S^{\prime}=\left\{\mathfrak{p}_{2}^{(1)}\right\}$ et $T^{\prime}=$ $\left\{\mathfrak{p}_{2}^{(2)}, \mathfrak{p}_{4}\right\}$. Un calcul montre que le morphisme de $\mathbb{Z}_{2}$-modules $\varphi_{S^{\prime}}:=$ $\mathbb{Z}_{2} \otimes E_{\mathrm{K}}^{T^{\prime}} \rightarrow U_{\mathfrak{p}_{2}^{(1)}}^{1}$ est injectif. Par conséquent, $\mathrm{rk}_{\mathbb{Z}_{2}} G_{S^{\prime}}^{T^{\prime}, a b}=7-(4+2)=1$. Il existe une unique $\mathbb{Z}_{2}$-extension $\mathrm{K}_{\infty}^{\prime}$ de $\mathrm{K}$ contenue dans $\mathrm{K}_{S}$ et dans laquelle, les places $\mathfrak{p}_{2}^{(2)}$ et $\mathfrak{p}_{4}$ sont totalement décomposées. En fait, on a : $\operatorname{Gal}\left(G_{S^{\prime}}^{T^{\prime}} / \mathrm{K}\right) \simeq \mathbb{Z}_{2}$

La proposition 5.3 indique que pour cette $\mathbb{Z}_{2}$-extension $\mathrm{K}_{\infty}^{\prime} / \mathrm{K}$, l'invariant $\mathrm{r}_{S}^{\prime}$ du $\mathbb{Z}_{2}\left[\left[\mathrm{Gal}\left(\mathrm{K}_{\infty}^{\prime} / \mathrm{K}\right)\right]\right]$-module $\operatorname{Gal}\left(\mathrm{K}_{S} / \mathrm{K}_{\infty}^{\prime}\right)^{a b}$ est au moins 1 . Calculons sa valeur exacte : Le corps $K_{1}^{\prime}=K\left(\sqrt{-\varepsilon_{1} \cdot \varepsilon_{2} \cdot \varepsilon_{3} \cdot \theta}\right)$ est le premier étage de $\mathrm{K}_{\infty}^{\prime} / \mathrm{K}$. On vérifie ensuite que $\rho_{T}^{\prime S}=\mathrm{r}_{T}^{\prime S}=0$. Ainsi, la proposition 5.3 indique que l'invariant $\mathrm{r}_{S}^{\prime}$ est égal à 1 .

En résumé, le pro-2-groupe $\mathrm{Gal}\left(\mathrm{K}_{S} / \mathrm{K}_{\infty}^{\prime}\right)$ n'est pas libre. Il est de dimension cohomologique 2 .

\section{Bibliographie}

[1] C. Batut. K. Belabas, H. Cohen, M. Olivier, User's guide to PARI-GP. A2X, Université Bordeaux I, 1999.

[2] N. Boston, Explicit Galois groups of infinite p-extensions unramified outside p, preprint 2003.

[3] N. Boston, Some cases of the Fontaine-Mazur conjecture. Journal of Number Theory 42 (1992), 285-291.

[4] N. Boston, Some cases of the Fontaine-Mazur conjecture II. Journal of Number Theory 75 (1999), 161-169.

[5] J.-M. Fontaine, B. MAZur, Geometric Galois representations. Elliptic curves. modular forms and Fermat's last theorem, Internat. Press, Cambridge, MA, 1995.

[6] R. Gillard, Fonctions L p-adiques des corps quadratiques imaginaires et de leurs extensions abéliennes. Crelle 358 (1985), 76-91.

[7] G. Gras, Class Field Theory. SMM, Springer 2003.

[8] G. Gras, Groupe de Galois de la p-extension abélienne p-ramifiée maximale d'un corps de nombres. Crelle 333 (1982), 86-132. 
[9] G. GRAS, Plongements kummériens dans les $\mathbb{Z}_{p}$-extensions. Compositio Math. 55 (1985), 383-396.

[10] G. Gras. J.-F. JAulent, Sur les corps de nombres réguliers. Math. Z. 202 (1989), 343-365.

[11] K. HABERLAND, Galois cohomology of algebraic number fields, With two appendices by Helmut Koch and Thomas Zink. VEB Deutscher Verlag der Wissenschaften, Berlin, 1978.

[12] F. HAJIR, C. MAIRE, Extensions of number fields with ramification of bounded depth. International Math. Research Notices 13 (2002), 667-696.

[13] H. Hasse, Number Theory. Classics in Mathematics, Springer, 1980.

[14] J.-F. Jaulent, L'arithmétique des l-extensions. Publications Math. de Besançon, 1986.

[15] J.-F. Jaulent, C. MAIRE, Sur les invariants d'Iwasawa des tours cyclotomiques. Canadian Math. Bulletin 46 (2003), 178-190.

[16] J.-F. JAUlent, T. NGUYen QuANG Do, Corps p-rationnels, corps réguliers et ramification restreinte. J. Théorie des Nombres de Bordeaux 5 (1993), 343-363.

[17] J.-F. Jaulent. O. SAuzet, Pro-l-extensions de corps de nombres l-rationnels. Jour. Number Theory 65 (1997), 240-267.

[18] H. КосH, Galoissche Theorie der p-Erweiterungen. VEB, Berlin, 1970.

[19] J. LABUTE, Mild pro-p-groups and Galois groups of p-extensions of $\mathbb{Q}$. J. Reine Angew. Math., à paraître.

[20] C. MAIRE, On the $\mathbb{Z}_{l}$-rank of abelian extensions with restricted ramification. Journal of Number Theory 92 (2002), 376-404.

[21] A. MovahHedi, Sur les p-extensions des corps p-rationnels. Math. Nach. 149 (1990), 163176.

[22] A. Movahhedi, T. Nguyen Quang Do, Sur l'arithmétique des corps de nombres prationnels. Sém. Théorie des Nombres, Paris (1987/89), Prog. Math 81, 155-200.

[23] J. NeukirCh, A. SChmidt, K. WingBerg, Cohomology of Number Fields. Grundlehren 323, Springer-Verlag 2002.

[24] T. NGUYen QUang Do, Formations de classes et modules d'Iwasawa. Number Theory, Noordwijkerhout 1983, Lecture Notes in Math. 1068, 167-185.

[25] A. Schmidt, On the relation between 2 and $\infty$ in Galois Cohomology of Number Fields. Compositio Math. 133 (2002), 267-288.

[26] A. Schmidt, Circular sets of prime numbers and p-extensions of the rationals, preprint, 2005.

[27] A. Schmidt, Bounded defect in partial Euler characteristics. Bull. London Math. Soc. 28 (1996), 463-464.

[28] L. SchNePS, On the $\mu$-invariant of $p$-adic L-functions attached to elliptic curves with complex multiplication. Journal of Number Theory 25 (1987), 20-33.

[29] J.-P. SERre, Galois Cohomology. Lecture Notes in Math., Springer-Verlag, Berlin, 1994.

[30] J.-P. Serre, Corps locaux. Publications de l'Université de Nancago, Hermann, Paris, 1968.

[31] L.C. Washington, Introduction to cyclotomic fields. GTM 83, Springer 1997.

[32] K. WingBerg, Galois groups of number fields generated by torsion points of elliptic curves. Nagoya Math. J 104 (1986), 43-53.

\author{
Christian MAIRE \\ GRIMM \\ Université Toulouse le Mirail \\ 5 , allées A. Machado \\ 31058 Toulouse cédex \\ E-mail : maire@univ-tlse2.fr \\ $U R L:$ http://www.univ-tlse2.fr/grimm/maire
}

